DESY 08-207

\title{
Collider constraints on interactions of dark energy with the Standard Model
}

\author{
Philippe Brax ${ }^{1}$, Clare Burrage ${ }^{2}$, Anne-Christine Davis ${ }^{3}$, \\ David Seery ${ }^{3}$ and Amanda Weltman ${ }^{3,4}$ \\ ${ }^{1}$ Institut de Physique Théorique, CEA, IPhT, CNRS, URA2306, F-91191 \\ Gif-sur-Yvette cédex, France \\ 2 Theory Group, Deutsches Elektronen-Synchrotron DESY, D-22603, Hamburg, \\ Germany \\ ${ }^{3}$ Department of Applied Mathematics and Theoretical Physics \\ Centre for Mathematical Sciences, University of Cambridge, \\ Wilberforce Road, Cambridge, CB3 0WA, United Kingdom \\ ${ }^{4}$ Department of Mathematics and Applied Mathematics, University of Cape Town, \\ Private Bag, Rondebosch, South Africa, 7700 \\ E-mail: djs61@cam.ac.uk
}

\begin{abstract}
We study models in which a light scalar dark energy particle couples to the gauge fields of the electroweak force, the photon, $Z$, and $W^{ \pm}$bosons. Our analysis applies to a large class of interacting dark energy models, including those in which the dark energy mass can be adjusted to evade fifth-force bounds by the so-called "chameleon" mechanism. We conclude that - with the usual choice of Higgs sectorelectroweak precision observables are screened from the indirect effects of dark energy, making such corrections effectively unobservable at present-day colliders, and limiting the dark energy discovery potential of any future International Linear Collider. We show that a similar screening effect applies to processes mediated by flavour-changing neutral currents, which can be traced to the Glashow-Iliopoulos-Maiani mechanism. However, Higgs boson production at the Large Hadron Collider via weak boson fusion may receive observable corrections.
\end{abstract}

Keywords: Dark energy theory, Weak interactions beyond the Standard Model, Cosmology of theories beyond the Standard Model 


\section{Introduction}

The emergence of cosmology as a data-driven science in the late 1990s enabled our theories of the universe to be promoted from mostly speculation to meaningful quantitative investigation. Although many components of what now forms the standard "concordance" $\Lambda$ CDM cosmology had been proposed prior to the quantitative revolution and were found to be consistent with experiment, among the more surprising revelations was the emergence of a new scale at around $10^{-3} \mathrm{eV}$, associated with an apparent acceleration of the cosmological expansion. The properties of Nature at this scale have been accessible since the earliest days of particle physics, and our models of microscopic processes at these energies are now very well tested. It was therefore surprising to discover that this hitherto mundane scale was to be associated with an exotic species of matter with energy density $\Lambda \sim\left(10^{-3} \mathrm{eV}\right)^{4}$ and equation of state $p \approx-\rho$.

The microphysics associated with this energy density remains unknown. The most parsimonious interpretation of the data requires only Einstein's "cosmological term," which we now know to be degenerate with the aggregate effect of quantum vacuum fluctations. At a mass scale $M$ these contribute a cosmological effect of order $M^{4}$. Consequently, if we take our well-tested quantum-mechanical theories of physics seriously even at comparatively modest scales (up to $M_{\mathrm{EW}} \sim 100 \mathrm{GeV}-1 \mathrm{TeV}$ ) then without a remarkable cancellation we encounter a serious disagreement with the data. An alternative interpretation is to suppose that unknown physics renders the quantum zero-point energy negligible or unobservable. If this is the case, it is possible that our present phase of acceleration is driven by the potential energy associated with some scalar field. This field would have to be very light on large scales in our present vacuum, with mass of order $H_{0} \sim 10^{-33} \mathrm{eV}$, but it might evade the stringent bounds associated with long-range forces mediated by light bosons if its mass could be adjusted to be large in regions of high average density. Theories of this type were proposed by Khoury \& Weltman [1, 2], who called such fields "chameleonic" in view of their ability to vary their properties depending on the environment $\dagger$

The chameleon property means that models involving these fields can give rise to successful acceleration at late times [5], while remaining consistent with known constraints on long-range physics. Such models are attractive for another reason, because the requirement that the field can respond to local variations in the density of bulk matter means that couplings to Standard Model states are mandatory. Chameleonic fields are therefore constrained by precision measurements of the early universe - in particular, observations of Big Bang Nucleosynthesis ('BBN') and the redshift of recombination [5, 6]. As the universe cools the background dark energy field remains fixed in the minimum of its potential, whose location slowly drifts. The result is a variation in the mass of any particle to which dark energy is coupled. However, acceptable models are constructed in such a way that only small changes in particle mass can be expected, and therefore the constraints from observations such as BBN are

$\dagger$ For earlier work, see Refs. [3, 4]. 
rather weak. Interesting bounds have also been obtained from a variety of astrophysical and terrestrial processes [7, 8, 9, 10, 11, 12, 13, 14, 15].

These couplings also imply the existence of an interesting collider phenomenology. With the aim of complementing the cosmological and astrophysical tests, our purpose in this paper is to take the first steps towards understanding the implications of dark energy corrections for Standard Model processes which can be observed at present and future particle colliders. A related study has been performed by Kleban \& Rabadan [16].

What form would these corrections take? We expect that the dark energy scalar is not charged under any of the usual gauge quantum numbers associated with the Standard Model. Its couplings to Standard Model states are therefore unrestricted by considerations of gauge invariance. Nevertheless, because bulk mass in the macroscopic world is dominated by hadrons it seems unavoidable for a chameleonic scalar to couple to those degrees of freedom charged under QCD, namely the quarks and gluons. Unfortunately, hadron interactions in QCD are non-perturbative in nature and are difficult to study. It is less obvious that the dark energy is obliged to couple to degrees of freedom charged under the electroweak $S U(2) \times U(1)$ gauge symmetry, but if it does then one might imagine that such interactions would offer a more tractable probe of the theory than the complicated colour physics of QCD. Our purpose in this work is to study the comparatively clean experimental signatures which arise at low energy from the existence of couplings between dark energy and Standard Model states which carry electroweak quantum numbers.

Interactions between a scalar dark energy species and the electroweak sector need not be harmless. For example, variation in the dark energy vacuum expectation value could lead to a shifting fine-structure constant or loss of conservation of electric charge [17, 18]. From the perspective of collider phenomenology, there is another serious difficulty: fundamental scalar fields are well-known to depend sensitively on the details of physics in the ultra-violet. If Standard Model particles can radiate into light chameleon states while participating in some measurable process, then we must allow for the possibility of significant corrections to observable Standard Model reactions. Indeed, it is a serious question whether any dark energy model of this type can be compatible with existing data. It is also important to understand whether we should expect dramatic signals at impending high-precision experiments such as the Large Hadron Collider (LHC) at the European Organization for Nuclear Research (CERN) or at a proposed future International Linear Collider.

In this paper, we study the effect of such radiative corrections. Our results apply to models of chameleon dark energy, and also to alternatives such as coupled quintessence, or any beyond-the-Standard-Model scalar species which is light in the laboratory environment. Similar issues have been addressed previously by Einhorn \& Wudka [19], who determined the criteria for heavy scalar particles to be screened. However, our results are not contained in their analysis because the scalar particles which can cause successful cosmological acceleration must ordinarily be very light in 
the laboratory environment compared to the electroweak scale, with masses of order $\lesssim 10^{-8} \mathrm{eV}$ or lighter. It is the effect of highly suppressed couplings in the laboratory which allows such particles to have evaded detection, rather than the significant energy cost of producing them in collisions.

In $₫ 2.1$ we give a brief summary of ultra-violet effects in scalar field theories, before going on to review the formalism used to study corrections to electroweak precision observables (\$3). In 93.1 we study corrections to the width of the $\mathrm{Z}$ boson (a tree-level effect), and show that it leads to a weak constraint. In $\$ 3.2$ we identify a class of loop effects which lead to stronger constraints, the so-called "oblique" corrections. The key quantities we require to compute them are the vacuum polarizations of the $W^{ \pm}, Z$ and $\gamma$ bosons, which are obtained in $\$ 4$. In $\$ 4.2$ we interpret these vacuum polarizations in terms of an effective Lagrangian which makes their physical content transparent. In \$5 we discuss our findings and indicate how our results could be extended to a larger zoology of processes, including so-called flavour-changing neutral currents. In particular, in \$5.1 we discuss the conditions under which the largest corrections are "screened," meaning that they do not enter in any measurable relationship between observables. It is only when screening occurs that the model is automatically compatible with the simplest predictions of the Standard Model. In $\$ 5.2$ we determine the constraints which can be obtained from data obtained by present-day colliders, and discuss the role of future hadron-hadron or $e^{+} e^{-}$colliders. Finally, in $\$ 6$ we state our conclusions. Some technical details are collected in two Appendices.

We choose units throughout such that $\hbar=c=1$. Our metric convention is $(-,+,+,+)$, so that on-shell particles have negative invariant momenta. Spacetime indices are denoted by lower-case Latin indices $\{a, b, c, \ldots\}$, and we label the species of vector bosons by upper-case indices $\{A, B, C, \ldots\}$.

\section{Electroweakly interacting dark energy}

\subsection{Ultra-violet effects}

The problem of sensitivity to ultra-violet effects is universal in any theory of scalar fields. While it is an obstacle for model-building, UV sensitivity can be exploited as a tool to probe the theory at energies much higher than those which can physically be realized in particle accelerators. An important example of this occurs in the Higgs sector of the Standard Model, which has many parallels with the case of interacting dark energy. For this reason we digress to give a brief discussion of the Higgs case, before returning to dark energy in $\$ 2.2$.

All particles which gain their mass via the Higgs mechanism are entitled to radiate into Higgs states, and in consequence it was pointed out long ago by Veltman that electroweak quantities can receive large Higgs contributions, up to some scale above which radiation is suppressed. This scale is presumably determined by a more complete theory of microscopic interactions, of which the Standard Model is an effective low 
energy limit. The Standard Model including a Higgs sector is precisely renormalizable, but if the Higgs is decoupled from the theory by taking its mass to infinity, $M_{H} \rightarrow \infty$, we should recover the divergences of the Higgsless case. One can therefore think of $M_{H}$ as a soft effective cutoff corresponding to the scale of new physics [20]. Any large Higgs contributions must appear experimentally as deviations from the tree-level expectation, which can be summarized in terms of Veltman's " $\rho$-parameter." In principle, this could receive corrections from the Higgs sector of the form

$$
\rho \equiv \frac{M_{W}^{2}}{M_{Z}^{2} \cos ^{2} \theta}=1+a_{0} g^{2} \frac{M_{H}^{2}}{M_{Z}^{2}}+a_{1} g^{2} \ln \frac{M_{H}^{2}}{M_{Z}^{2}}+\cdots,
$$

where $a_{0}$ and $a_{1}$ are pure numbers which must be calculated, $g$ is a coupling constant, and '...' denotes the effect of higher-order radiative corrections which we have neglected. The current experimental constraint is $\rho=1.0004_{-0.0004}^{+0.0008}$ [21], so if $a_{0} \neq 0$ one would obtain extremely stringent constraints on $M_{H}$. Unfortunately, in the Standard Model it turns out that $a_{0}=0$ [22, 23], leading to a considerably weaker bound $M_{H} \lesssim 215 \mathrm{GeV}$. This effect occurs in all Standard Model observables and has become known as the screening theorem, because it protects low-energy observations from the effect of coupling to a large phase space of scalar Higgs states. It has been shown that the screening phenomenon extends to all orders in the loop expansion in the limit $M_{H} \rightarrow \infty$ [24, 25, 19].

The same principles apply to any light scalar field. What happens if Standard Model particles are permitted to radiate into dark energy states? In the laboratory environment where the $W^{ \pm}$and $Z$ masses can be measured, the dark energy quanta are typically light. In this case, we must expect contributions to electroweak observables of the form described by Eq. (1), with the Higgs mass $M_{H}$ replaced by whatever scale $M$ determines the size of the phase space of available states, and the coupling $g^{2}$ replaced by whatever quantity sets the interaction strength of dark energy with ordinary matter, which is typically a number of order $M_{Z}^{2} / M^{2}$. It then becomes extremely significant whether dark energy exhibits a similar screening effect, for if $a_{0} \neq 0$ then $\rho$ will generically receive corrections of $\mathrm{O}(1)$. Such large corrections could easily lead to an unacceptable conflict with precision electroweak data. On the other hand, if the dark energy does exhibit screening then the corrections to $\rho$ are roughly of order $\mathrm{O}\left[\left(M_{Z} / M\right)^{2} \ln M^{2} / M_{Z}^{2}\right]$ and are therefore very small for any phenomenologically reasonable choice of $M$.

We would like to emphasize that there is no reason of principle for the Higgs or any other scalar species to exhibit this sort of radiative screening. In the Higgs sector, a so-called "custodial" global $S U(2)$ symmetry becomes exact in the limit where the hypercharge gauge coupling $g_{1}$ vanishes [26], which guarantees equality of the vector boson masses, but does not guarantee screening [20] In the absence of any specific reason to think otherwise, one must imagine that a generic scalar field theory interacting

$\dagger$ In their proof that the Higgs exhibits radiative screening to all orders in the loop expansion, Einhorn \& Wudka made essential use of the $S U(2)$ custodial symmetry [25]. However, although the existence of this symmetry is necessary, it is not sufficient. An integral part of of Einhorn \& Wudka's argument consists of a power-counting procedure entirely unconnected with the custodial symmetry, which determines where the leading divergences can appear as $M_{H} \rightarrow \infty$. 
with the electroweak sector would contribute to Eq. (11) with $a_{0} \neq 0$. Although it may be possible to fine-tune a model of this type to be consistent with precision electroweak observations, this solution would be highly unattractive. Indeed, one would have traded an unappealing fine-tuning in the cosmological constant for a fine-tuning in the scalar model intended to replace it, and little would have been gained.

\subsection{The interaction Lagrangian}

We will choose to work with a theory of the broken phase of the electroweak force in which the photon and the massive vector bosons $W^{ \pm}$and $Z$ interact with a single dark energy scalar $\chi$ according to the action

$$
\begin{aligned}
S=-\frac{1}{4} \int \mathrm{d}^{4} x\{ & 2 B(\beta \chi)\left(\partial^{a} W^{+b}-\partial^{b} W^{+a}\right)\left(\partial_{a} W_{b}^{-}-\partial_{b} W_{a}^{-}\right)+4 m_{W}^{2} B_{H}\left(\beta_{H} \chi\right) W^{+a} W_{a}^{-} \\
& +B(\beta \chi)\left(\partial^{a} Z^{b}-\partial^{b} Z^{a}\right)\left(\partial_{a} Z_{b}-\partial_{b} Z_{a}\right)+2 m_{Z}^{2} B_{H}\left(\beta_{H} \chi\right) Z^{a} Z_{a} \\
& \left.+B(\beta \chi)\left(\partial^{a} A^{b}-\partial^{b} A^{a}\right)\left(\partial_{a} A_{b}-\partial_{b} A_{a}\right)\right\}
\end{aligned}
$$

where $W_{a}^{ \pm}$and $Z_{a}$ are the gauge fields associated with the $W^{ \pm}$and $Z$, respectively, and $A_{a}$ is the gauge field associated with the photon. Eq. (2) should be thought of as an effective Lagrangian valid after integrating out the Goldstone modes of the Higgs, as emphasized by Burgess \& London [27, 28] following earlier work in Refs. [29, 30]. Only invariance under the electromagnetic $U(1)$ gauge group is required.

The quantities $m_{W}$ and $m_{Z}$ are the Lagrangian parameters corresponding to the mass of the $W^{ \pm}$and $Z$, which are related via a renormalization prescription to the physical masses $M_{W}$ and $M_{Z}$. In addition, we have introduced two arbitrary functions $B(\beta \chi)$ and $B_{H}\left(\beta_{H} \chi\right)$ which describe how the scalar $\chi$ couples to the gauge boson kinetic and mass terms. These couplings are associated with mass scales $M \equiv \beta^{-1}$ and $M_{H} \equiv \beta_{H}^{-1}$ (not necessarily identica $\ddagger$ ) which control the relative strength of the interaction between dark energy and the weak gauge bosons, and between dark energy and the Higgs field respectively.

Throughout this paper, we assume that the dark energy quanta $\chi$ have some fixed mass $M_{\chi}$, which is not subject to renormalization. This is tantamount to treating the entire scalar sector as an effective field theory, in which quantum effects have already been included, and for which we only wish to assess the influence of radiative corrections on the bare electroweak sector. This is appropriate for a phenomenological model such as a chameleon, which need not be a fundamental particle in its own right, but rather may represent the collective effect of degrees of freedom at high energy which have been integrated out of the theory. In any such effective field theory it is difficult to maintain light scalar masses because quantum corrections will typically renormalize these to the scale of the cutoff unless they are protected by a symmetry. This difficulty afflicts all $\ddagger$ Note that $M_{H}$ is not the Higgs mass, which was discussed in $₫ 2.1$ but does not appear in the remainder of this paper. 

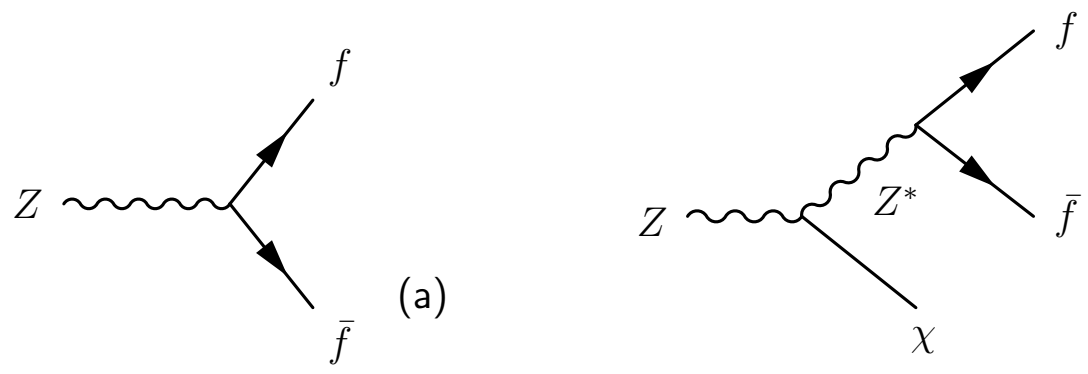

Figure 1. Contributions to the decay width of the neutral $Z$ boson. In (a), an onshell $Z$ decays to a fermion-antifermion pair $f \bar{f}$. In (b), the decay is precipitated by emission of a dark energy particle, $\chi$, leaving the original $Z$ in an off-shell excited state which subsequently decays to $f \bar{f}$. If the final-state $\chi$ is not observed, these processes cannot be distinguished and therefore both contribute to the decay width into $f \bar{f}$.

particulate theories of dark energy equally, and we have nothing new to contribute to this debate.

The coupling functions $B$ and $B_{H}$ are unknown, although they will be subject to certain restrictions if we wish the dark energy field to exhibit an acceptable chameleon phenomenology. We will not impose any such restrictions, except to observe that the coupling functions for the $W^{ \pm}, Z$ and $\gamma$ kinetic terms must be the same if Eq. (2) is to descend from an unbroken gauge-invariant theory of $S U(2) \times U(1)$ at higher energies. Moreover, the coupling functions multiplying the mass terms must be the same if we suppose that the $W^{ \pm}$and $Z$ obtain their masses via spontaneous symmetry breaking, and that the Higgs sector consists of a minimal $S U(2)$ doublet. Since we wish to retain both these phenomenological successes of the Standard Model, we are left with at most two free coupling functions. In many cases, however, we expect that Eq. (2) will not have a UV completion unless these couplings are the same, because the longitudinal polarizations of the $Z$ and $W^{ \pm}$are associated with Goldstone modes of the Higgs.

\section{Electroweak precision observables}

\subsection{Constraints from $Z$ decay}

Let us first consider corrections where some dark energy quanta are present in the final state. These corrections can be considered as a form of "dark energy bremsstrahlung". Since the final-state dark energy particles escape the detector and are not observed, such reactions look like extra contributions to the cross-section for the corresponding bare Standard Model process. Among the best-measured of these is the width for $Z$ decay, depicted for decay into a fermion-antifermion pair $f \bar{f}$ of common mass $M_{f}$ with and without dark energy dressing in Figs. 1(a) and (b) respectively. In the dressed process (b) the on-shell 4-momentum of one outgoing fermion (which we label ' 2 ' by convention) is fixed by conservation of 3-momentum. The energy of the other fermion is determined by energy conservation in terms of a quadratic equation to be given below. We show in 
Appendix A that the differential contribution to the $\mathrm{Z}$ decay width from emission of a single dark energy particle of energy $E_{\chi}$ into a solid angle $\mathrm{d} \Omega_{\chi}$ satisfies

$\frac{\mathrm{d} \Gamma(Z \rightarrow \chi f \bar{f})}{\Gamma(Z \rightarrow f \bar{f})}=\frac{\bar{B}^{\prime 2}}{(2 \pi)^{3}} \frac{M_{Z}^{2}}{M^{2}} \mathrm{~d} \hat{E}_{\chi} \mathrm{d} \Omega_{\chi} \frac{\sqrt{\hat{E}_{\chi}^{2}-y^{2}} \sqrt{\hat{E}_{1}^{2}-x^{2}}}{J\left(1-\hat{E}_{\chi}-\hat{E}_{1}\right)\left(1+\hat{r}^{2}\right)^{2}} \frac{\mathcal{M}_{\chi f \bar{f}}}{\mathcal{M}_{f \bar{f}}}$,

where $M=\beta^{-1}$ is the dark energy coupling scale, and $x$ and $y$ are defined by

$$
\begin{aligned}
& x \equiv \frac{M_{f}^{2}}{M_{Z}^{2}} \\
& y \equiv \frac{M_{\chi}^{2}}{M_{Z}^{2}} .
\end{aligned}
$$

The outgoing dark energy scalar is taken to have 3-momentum q. We introduce dimensionless "hatted" energies and momentum according to the rules

$$
\begin{aligned}
& \hat{E}_{i}=\frac{E_{i}}{M_{Z}} \\
& \hat{\mathbf{q}}=\frac{\mathbf{q}}{M_{Z}} .
\end{aligned}
$$

where $i \in\{\chi, 1,2\}$. The quantity $\hat{r}^{2}$ measures the degree to which the intermediate $Z^{*}$ is off-shell, and satisfies

$$
\hat{r}^{2} \equiv-1+2 \hat{E}_{\chi}-y^{2} .
$$

It is equal to -1 for an intermediate $Z$ which is precisely on-shell, although in this limit the finite width of the $Z$ cannot be ignored. The energy $\hat{E}_{1}$ must be a solution of the quadratic equation

$$
\begin{gathered}
\hat{E}_{1}^{2}\left\{\cos ^{2} \theta\left(\hat{E}_{\chi}^{2}-y^{2}\right)-\left(1-\hat{E}_{\chi}^{2}\right)\right\}+\hat{E}_{1}\left(1-\hat{E}_{\chi}\right)\left(1+y^{2}-2 \hat{E}_{\chi}\right) \\
=\frac{1}{4}\left(1+y^{2}-2 \hat{E}_{\chi}\right)^{2}+x^{2}\left(\cos ^{2} \theta\right)\left(\hat{E}_{\chi}^{2}-y^{2}\right)
\end{gathered}
$$

where $\theta$ is the angle between $\mathbf{q}$ and the 3 -momentum of fermion 1 . Although two solutions for $\hat{E}_{1}$ exist, one is always spurious. The solutions change roles at $\theta=\pi / 2$. Moreover, $J$ is a Jacobian arising from fixing $\hat{E}_{1}$ to be a solution of Eq. (9). It is defined by

$$
J=\left|1+\hat{E}_{1} \frac{1+\left(\hat{E}_{\chi}^{2}-y^{2}\right)^{1 / 2}\left(\hat{E}_{1}^{2}-x^{2}\right)^{-1 / 2} \cos \theta}{1-\hat{E}_{\chi}-\hat{E}_{1}}\right| .
$$

The matrix element $\mathcal{M}_{f \bar{f}}$ satisfies

$$
\mathcal{M}_{f \bar{f}}=\sqrt{1-4 x^{2}}\left\{6 g_{L} g_{R} x^{2}+\left(g_{L}^{2}+g_{R}^{2}\right)\left(1-x^{2}\right)\right\},
$$

where $g_{L}$ and $g_{R}$ are the left- and right-handed couplings of the fermion species to the $Z$; and $\mathcal{M}_{\chi f \bar{f}}$ is a complicated function whose form is determined in Appendix A and which can be read off from Eqs. (A.9)-(A.10) or Eqs. (A.14)-(A.15). After integrating over $\hat{E}_{\chi}$ and the solid angle $\Omega_{\chi}$, Eq. (3) determines the cross-section for any dressed 
process in terms of the bare standard model cross-section. In a generic model without fine-tuning, for which $\bar{B}^{\prime} \sim 1$, this rate takes the form

$$
\frac{\Gamma(Z \rightarrow \chi f \bar{f})}{\Gamma(Z \rightarrow f \bar{f})}=\frac{1}{16 \pi^{3}} \frac{M_{Z}^{2}}{M^{2}} I_{\chi f \bar{f}}
$$

and $I_{\chi f \bar{f}}$ is found to be numerically of order $I_{\chi f \bar{f}} \approx 0.2$ for a wide range of fermion masses and couplings. The width of the $Z$ into visible particles is predicted to be $\Gamma_{Z}=2.4952 \mathrm{GeV}$ within the Standard Model, with a small theoretical error. Its measured value is $\Gamma_{Z}=(2.4952 \pm 0.0023) \mathrm{GeV}$ [21], implying that any enhancement due to dark energy will be compatible with observation only if $M \gtrsim 0.66 M_{Z} \sim 60 \mathrm{GeV}$. Moreover, our neglect of the $Z$ width means that this is a conservative over-estimate. Thus, under the very mild constraint $M \gtrsim M_{Z}$ it seems clear that there will be no disagreement with the data. Processes similar to Fig. 1(b), but with emission of more than one dark energy particle into the final state, are suppressed by extra powers of $\left(M_{Z} / M\right)^{2}(2 \pi)^{-3}$.

Dark energy bremsstrahlung could have consequences beyond enhancements to decay widths and cross-sections of the sort calculated above. Soft bremsstrahlung effects could be significant in QCD if they initiated jet formation by destabilizing quarks or gluons, or if their aggregate effect could be resolved by partons participating in a sufficiently hard collision. However, such effects are likely to be important only if the dark energy couples at a very low scale. We can estimate that the S-matrix element for any bremsstrahlung event should controlled by the square of the single-chameleon coupling constant, of order $M_{f} / M$ for a fermion of mass $M_{f}$, and a phase space factor of order $\ln s / M_{\chi}^{2}$, where $s \approx M_{\mathrm{EW}}^{2}$ is the usual Mandelstam variable and $M_{\chi} \lesssim 10^{-8} \mathrm{eV}$ is the dark energy mass in the beam pipe [31, 32]. The logarithm is roughly of order $10^{2}$. A significant effect can occur if the product $\left(M_{f} / M\right)^{2} \ln M_{\mathrm{EW}}^{2} / M_{\chi}^{2} \sim 1$, but unless the dark energy scalar is very light this combination is generally negligible whenever the coupling scale $M$ is modestly larger than the mass of the fermion species in question, of order $M \gtrsim 10^{2} M_{f}$.

\subsection{Oblique corrections}

In addition to bremsstrahlung processes, the perturbation theory constructed from Eq. (2) describes processes by which Standard Model particles may radiate into an intermediate state containing an arbitrary number of dark energy quanta. If we exclude reactions in which dark energy particles are present in the initial or final state then all such processes are built out of interactions which are already present in the bare Standard Model. To study them we should begin with a given Standard Model reaction, exemplified for the case of $2 \rightarrow 2^{\prime}$ scattering of light fermions in Fig. 2(a), and account for the effect of dark energy activity. This activity can naturally be divided into three categories, corresponding to Figs. 2(b)-(d).

In Fig. 2(b), dark energy loops dress each vertex in the bare reaction with socalled daisies, whereas in Fig. 2(c) dark energy quanta bridge between two different 


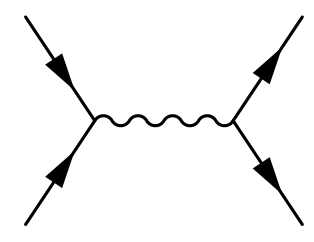

(a)

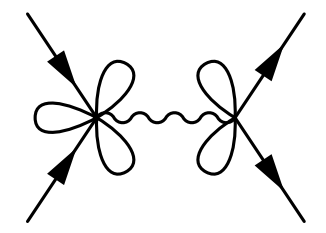

(b)

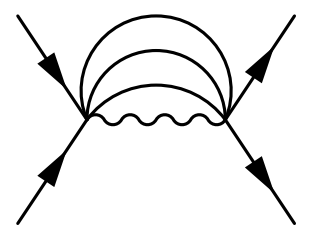

(c)

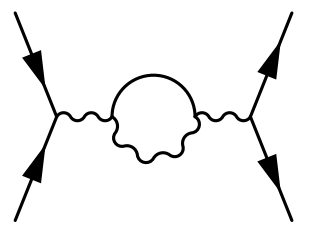

(d)

Figure 2. Classes of dark energy diagrams associated with Standard Model reactions, exemplified in the case of $2 \rightarrow 2^{\prime}$ fermion scattering. Solid lines with arrows represent fermions; wavy lines represent the gauge bosons of the electroweak force; and plain lines represent dark energy particles. The bare Standard Model process is given in (a). In (b), the vertices of the reaction are dressed by daisies which begin and end at the same vertex. In (c), dark energy quanta bridge between two different vertices. Corrections such as (b)-(c) which depend on the process under study (in this case, depending on the initial and final fermion species, and the identity of the exchanged boson) are called straight. On the other hand, corrections such as $(d)$ which are universal for all processes involving the exchange of a given species of vector boson are called oblique. (In principle there are also oblique corrections to the fermion species, but typically these do not contribute significantly to observable quantities.) In general, the dark energy correction to (a) consists of summing over all possible combinations of processes similar to (b)-(d).

vertices. More complicated bridges, including internal vertices which may themselves be dressed by daisies, can also be constructed $\downarrow$ Together with processes where one or more dark energy quantum appears in the final state, these are examples of so-called straight corrections which depend on the process which under consideration [33].

In contradistinction, Fig. 2(d) represents an example of an oblique correction, which involves intermediate dark energy states only in the interior of a gauge boson propagator. Once an oblique correction has been calculated for a given species of gauge field, it is universal for all processes involving exchange of that boson. In principle, these corrections are all equally important and for a general momentum transfer $q$ it is a complicated process to compute them. However, we argue in Appendix B that the daisies and bridges which constitute the straight corrections are momentum-

$\dagger$ Note, however, that we do not include loops in which the chameleon interacts with itself: as has been said, these are assumed to have been absorbed in the parameters of the dark energy model. 


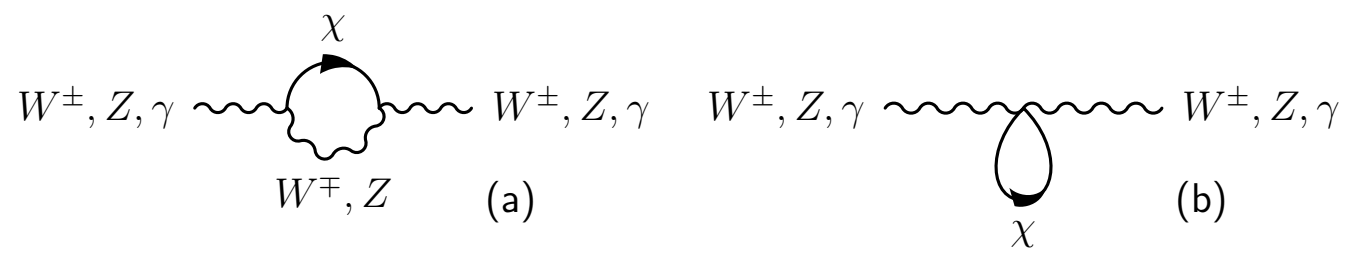

Figure 3. Processes contributing to the self-energy of the intermediate vector bosons $\gamma, W^{ \pm}$and $Z$. An initial vector boson state, represented by a wavy line, radiates into scalar quanta $\chi$ (represented by a solid line) which are eventually re-absorbed to yield a final state characterized by the same quantum numbers and momentum as the initial state.

independent up to terms of order $q^{2} / M^{2}$, where $M>M_{\mathrm{EW}}$ is a dark energy coupling scale characteristic of the fermion species which participate. Provided they are the same for all species, such momentum-independent terms can be absorbed in a renormalization of the Fermi constant, $G_{F}$, and are therefore unobservable. We have seen in \$3.1 that in any phenomenologically acceptable scenario we expect $M \gg M_{\mathrm{EW}}$, implying that the remaining contributions can be neglected in comparison with that of the oblique correction, Fig. 2(d), which is present at order $q^{2} / M_{\mathrm{EW}}^{2}$. Oblique corrections will therefore give the most stringent constraints if they turn out to require $M \gtrsim 100 \mathrm{GeV}$.

The effect of physics beyond the Standard Model has been studied by many authors, and is frequently dominated by oblique corrections. Peskin \& Takeuchi [33, 34] introduced a simple parametrization of them in terms of three quantities $S, T$ and $U$ which quantify the magnitude of corrections near zero momentum transfer $\ddagger$ but assumed that whatever new physics was responsible for modifying the properties of the gauge bosons was heavy. This assumption was later removed by Maksymyk, London \& Burgess [37, 38], who introduced new parameters $V, W$ and $X$ to quantify the significance of radiative corrections around the $Z$ resonance $\$$ In the remainder of this section, we briefly review the parametrization of oblique corrections in terms of $S, T$, $U, V, W$ and $X$.

The one-loop obliquely-corrected vector boson propagators are obtained by summing over an arbitrary number of insertions of the one-loop diagrams of Fig. 3 in the tree-level propagator. In unitarity gauge, where the three would-be Goldstone modes supplied by the Higgs doublet have been absorbed as longitudinal polarizations of the $W^{ \pm}$and $Z$, the tree-level propagator for each massive vector boson can be written

$$
\left\langle X_{A}^{a}\left(k_{1}\right) X_{B}^{\dagger b}\left(k_{2}\right)\right\rangle=-\mathrm{i}(2 \pi)^{3} \delta\left(k_{1}+k_{2}\right) \delta_{A B}\left(\eta^{a b}+\frac{k^{a} k^{b}}{m_{A}^{2}}\right) \Delta\left(k^{2}\right),
$$

where we have defined $k \equiv k_{1}=-k_{2}$ and the quantum field $X_{A}$ is built out of the creation and annihilation operators corresponding to a vector boson of species $A$ and mass $m_{A}$. The tree-level propagator function satisfies $\Delta^{-1}\left(k^{2}\right)=k^{2}+m_{A}^{2}$. The photon propagator can be written in an analogous form, with $m_{A} \mapsto 0$ in the function $\Delta\left(k^{2}\right)$

$\ddagger$ An alternative parametrization was proposed simultaneously by Altarelli \& Barbieri [35, 36].

$\S$ See also Refs. [39, 40]. 
and $m_{A}^{2} \mapsto-k^{2}$ in the tensor prefactor.

We define the sum of the one-particle-irreducible diagrams which connect an initialstate vector boson of species $A$ with a final-state vector boson of species $B$ and carrying momentum $k$ to be $i \Pi_{A B}^{a b}\left(k^{2}\right) /(2 \pi)^{4}$. Since the $Z$ and $\gamma$ are electrically neutral they are permitted to mix beyond tree-level, which would correspond to a non-zero vacuum polarization $\prod_{Z \gamma}^{a b}$. However, inspection of the interactions in Eq. (2) shows that Eq. (22) does not induce extra mixing and we can set $\Pi_{Z \gamma}^{a b}=0$.

With this simplification, the full propagator can be resummed using the SchwingerDyson equations. The result is that the propagator function $\Delta$ in Eq. (13) should be replaced by a resummed function $\Delta^{\prime}$, which for each species $A$ satisfies

$$
\Delta^{\prime}\left(k^{2}\right)=\frac{1}{k^{2}+m_{A}^{2}-\Pi_{A A}^{(0)}\left(k^{2}\right)},
$$

where we have written

$$
\Pi_{A B}^{a b}\left(k^{2}\right)=\eta^{a b} \Pi_{A B}^{(0)}\left(k^{2}\right)+k^{a} k^{b} \Pi_{A B}^{(2)}\left(k^{2}\right),
$$

and, for external states which consist only of light fermions of invariant mass-squared $M_{f}^{2}$. Eq. (14) is valid up to corrections of order $M_{f}^{2} / M_{W}^{2}$ which we neglect. Therefore, the quadratic term $\Pi_{A B}^{(2)}$ will not appear in the remainder of this paper, and to simplify notation we write all subsequent formulae in terms of the abbreviation $\Pi_{A B} \equiv \Pi_{A B}^{(0)}$.

\subsection{The $S, T, U, V$ and $W$ parameters}

In the absence of radiative corrections, the Standard Model entails the existence of simple relationships among the observables of the theory. Since there are three free quantities which parametrize the broken phase - the two gauge couplings $g_{1}$ and $g_{2}$, together with the Higgs vacuum expectation value - it is necessary to take three masses or couplings from experiment. Once this so-called 'input parameter set' has been selected, all other observables can be expressed in terms of the chosen three. In the electroweak sector it is conventional to choose the input parameter set to comprise the fine structure constant $\alpha$, the Fermi coupling $G_{F}$, and the $Z$ mass, $M_{Z}$, which are presently the best measured electroweak quantities.

With the inclusion of radiative corrections, the original simple relationships among observables are modified. Indeed, in order to match the precision with which accelerator experiments can measure electroweak parameters, it is usually necessary to include several orders of radiative corrections which arise purely within the Standard Model. It may happen that these corrections are insufficient to account for the deviation of all observables from their tree-level values. The remainder must be ascribed to new physics: it is only this contribution from new physics which we wish to attribute to the effect of a dark energy scalar species. The relevant observables other than $\left\{\alpha, G_{F}, M_{Z}\right\}$ are the mixing angle, $\theta_{\mathrm{W}}$, and the $W^{ \pm}$mass, $M_{W}$, together with any cross-sections or decay rates which can be written in terms of all these quantities. At tree level, $\theta_{\mathrm{W}}$ and $M_{W}$ 
are related to the input parameter set via the rules

$$
\begin{aligned}
& \sin ^{2} \theta_{\mathrm{W}}\left(1-\sin ^{2} \theta_{\mathrm{W}}\right)=\frac{\alpha}{16 \sqrt{2} \pi M_{Z}^{2} G_{F}}, \\
& M_{W}^{2}=M_{Z}^{2} \cos ^{2} \theta_{\mathrm{W}},
\end{aligned}
$$

where $\cos \theta_{\mathrm{W}}$ in Eq. (17) is to be computed from the solution to Eq. (16).

The physical mass of a single-particle state corresponding to a vector boson is given by the pole of Eq. (14), which renormalizes the Lagrangian parameter $m_{A}$. Therefore, the physical mass $M_{A}$ satisfies

$$
M_{A}^{2}=\tilde{M}_{A}^{2}\left(1-\frac{\Pi_{A A}\left(-M_{A}^{2}\right)}{M_{A}^{2}}\right),
$$

where we have introduced a useful notation in which a tilde, as in $\tilde{M}_{A}$, denotes the value taken by a quantity in the Standard Model without oblique corrections. At tree-level, $\tilde{M}_{A}^{2}$ is simply equal to $m_{A}^{2}$, but Eq. (18) continues to apply to leading order in radiative corrections even if we allow the vector boson masses to receive renormalizations from loops purely within the Standard Model. On the other hand, the Fermi constant $G_{F}$ is defined as the coupling of the charged-current interaction at zero momentum transfer and receives an oblique correction [33, 34]

$$
G_{F}=\tilde{G}_{F}\left(1+\frac{\Pi_{W W}(0)}{M_{W}^{2}}\right) .
$$

Likewise, the fine structure constant measures the electromagnetic interaction at zero momentum transfer and receives an oblique correction from the photon self-energy,

$$
\alpha=\tilde{\alpha}\left(1+\hat{\Pi}_{\gamma \gamma}(0)\right),
$$

where $\hat{\Pi}_{\gamma \gamma}\left(k^{2}\right) \equiv \Pi_{\gamma \gamma}\left(k^{2}\right) / k^{2}$. Eqs. (19) and (20) apply even if we allow $\tilde{G}_{F}$ and $\tilde{\alpha}$ to receive corrections from pure Standard Model loops. It follows that we can write

$$
\frac{s_{\mathrm{W}}^{2}}{\tilde{s}_{\mathrm{W}}^{2}}=1+\frac{\alpha}{4 s_{\mathrm{W}}^{2}\left(c_{\mathrm{W}}^{2}-s_{\mathrm{W}}^{2}\right)} S-\frac{\alpha c_{\mathrm{W}}^{2}}{c_{\mathrm{W}}^{2}-s_{\mathrm{W}}^{2}} T
$$

and

$$
\frac{M_{W}^{2}}{\tilde{M}_{W}^{2}}=1-\frac{\alpha}{2\left(c_{\mathrm{W}}^{2}-s_{\mathrm{W}}^{2}\right)} S+\frac{\alpha c_{\mathrm{W}}^{2}}{c_{\mathrm{W}}^{2}-s_{\mathrm{W}}^{2}} T+\frac{\alpha}{4 s_{\mathrm{W}}^{2}} U,
$$

where we have introduced the useful abbreviations $s_{\mathrm{W}} \equiv \sin \theta_{\mathrm{W}}$ and $c_{\mathrm{W}} \equiv \cos \theta_{\mathrm{W}}$, and the parameters $S, T$ and $U$ are defined by [33, 34, 37]|l]

$$
\frac{\alpha}{4 s_{\mathrm{W}}^{2} c_{\mathrm{W}}^{2}} S \equiv \frac{\Pi_{Z Z}(0)-\Pi_{Z Z}\left(-M_{Z}^{2}\right)}{M_{Z}^{2}}-\hat{\Pi}_{\gamma \gamma}(0),
$$

\| Certain terms in these expressions change sign depending on the choice of signature for the metric. Under reverse of sign convention (which gives the timelike convention widely used in particle physics, in comparison with the spacelike convention adopted in this paper), the formulae for $S, T$ and $U$ should be modified by reversing the sign of each mass-square $M_{A}^{2}$, together with extra signs for each explicit factor of $k^{2}$ or $\mathrm{d} k^{2}$. This explains the difference in signs between Eqs. (23)-(25) and the original references, which used the signature $(+,-,-,-)$. Note also that in theories where the $Z$ and $\gamma$ mix beyond tree-level, $S$ and $U$ receive extra contributions. For details, see Refs. [33, 34, 37]. 


$$
\begin{aligned}
& \alpha T \equiv \frac{\Pi_{Z Z}(0)}{M_{Z}^{2}}-\frac{\Pi_{W W}(0)}{M_{W}^{2}}, \\
& \frac{\alpha}{4 s_{W}^{2}}(U+S) \equiv \frac{\Pi_{W W}(0)-\Pi_{W W}\left(-M_{W}^{2}\right)}{M_{W}^{2}}-\hat{\Pi}_{\gamma \gamma}(0) .
\end{aligned}
$$

Experimentally observable quantities such as the Veltman $\rho$-parameter, Eq. (1), can be written in terms of $S, T$ and $U$.

Electroweak data is not limited to measurements of the $W^{ \pm}$and $Z$ masses and the mixing angle, but includes cross-sections and decay rates. The standard LSZ formula [41 implies that the first-order shifts from oblique corrections in these quantities can be obtained from their tree-level values together with appropriate multiplication by wave function renormalization factors $Z_{A}$, defined for each species of massive boson $A$ by the rule

$$
\mathrm{Z}_{A} \equiv 1+\left.\frac{\mathrm{d}}{\mathrm{d} k^{2}} \Pi_{A A}\left(k^{2}\right)\right|_{k^{2}=-M_{A}^{2}} .
$$

To take account of these factors, it is necessary to introduce two further parameters $V$ and $W$ [37, 39, 40]

$$
\begin{aligned}
& \left.\alpha V \equiv \frac{\mathrm{d}}{\mathrm{d} k^{2}} \Pi_{Z Z}\left(k^{2}\right)\right|_{k^{2}=-M_{Z}^{2}}-\frac{\Pi_{Z Z}(0)-\Pi_{Z Z}\left(-M_{Z}^{2}\right)}{M_{Z}^{2}}, \\
& \left.\alpha W \equiv \frac{\mathrm{d}}{\mathrm{d} k^{2}} \Pi_{W W}\left(k^{2}\right)\right|_{k^{2}=-M_{W}^{2}}-\frac{\Pi_{W W}(0)-\Pi_{W W}\left(-M_{W}^{2}\right)}{M_{W}^{2}} .
\end{aligned}
$$

Oblique dark energy corrections to all purely electroweak observables can be written in terms of $S, T, U, V$ and $W$.

These parameters have simple physical interpretations. $S$ is a measure of the difference between the wavefunction renormalization of the $Z$ boson and the photon, $\gamma$. In an interacting theory, a state prepared with definite particle content and momentum at some early time may not manifest the same content when probed at a later time because the particles may radiate into any other states to which they couple. The probability for this to occur is quantified by the wavefunction renormalization.

$T$ is a measure of the extra isospin breaking at zero momentum which is contributed by new physics. This difference manifests itself in the relative strength of the chargedand neutral-current interactions. The precise balance between these interactions may be upset by coupling to the dark energy scalar, but in the Standard Model with a minimal Higgs sector $T$ is unlikely to receive large corrections unless isospin symmetry is broken explicitly at tree level. Similarly, $U$ is a measure of the difference between the $W^{ \pm}$and $Z$ wavefunction renormalizations. Finally, $V$ and $W$ quantify the difference between the wavefunction renormalizations of the $Z$ and $W^{ \pm}$bosons, respectively, on the mass-shell, compared with zero momentum. In what follows, we will see this structure emerge explicitly from our analysis.

I In theories where the $Z$ and $\gamma$ can mix beyond tree-level, it is necessary to introduce a third new parameter, $X$. See Ref. 37. 


\section{Vector boson vacuum polarizations}

To evaluate the $S, T, U, V$ and $W$ parameters, one requires an explicit expression for the vector boson vacuum polarizations. In this section, we obtain the necessary self-energies by calculating the two one-loop diagrams in Fig. 3 .

\subsection{Feynman rules}

Our detailed information concerning the properties of the $W^{ \pm}$and $Z$ bosons comes mostly from the LEPII experiment, which created these particles abundantly in headon $e^{+} e^{-}$collisions. The $W^{ \pm} \mathrm{S}$ and $Z \mathrm{~s}$ synthesized in this way were produced at rest in the beam-pipe and spent their entire lifetime within its vacuum, before decaying into other particles which could subsequently be detected. In the environment of the beam-pipe, we can assume that the scalar field has a constant vacuum expectation value $\bar{\chi}$ together with small excitations $\delta \chi$. To obtain the one-loop vacuum polarization, it is necessary to describe the interactions of the $W^{ \pm}$and $Z$ to order $\delta \chi^{2}$. For interactions involving a $W^{+}$and $W^{-}$the relevant vertices are:

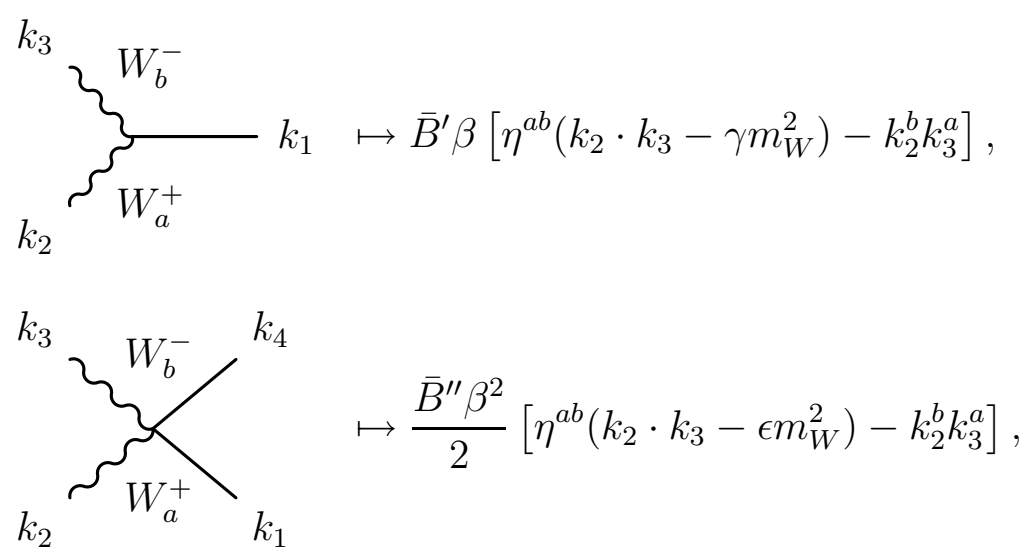

where $\bar{B}^{\prime} \equiv B^{\prime}(\beta \bar{\chi}), \bar{B}^{\prime \prime} \equiv B^{\prime \prime}(\beta \bar{\chi})$ together with equivalent definitions for $B_{H}$; the spacetime inner product is denoted $p \cdot q \equiv p^{a} q_{a}$ for any two four-vectors $p^{a}$ and $q^{a}$; and we have defined quantities $\gamma$ and $\epsilon$ according to the rules

$$
\begin{gathered}
\gamma \equiv \frac{\bar{B}_{H}^{\prime}}{\bar{B}^{\prime}} \frac{\beta_{H}}{\beta} \\
\epsilon \equiv \frac{\bar{B}_{H}^{\prime \prime}}{\bar{B}^{\prime \prime}} \frac{\beta_{H}^{2}}{\beta^{2}} .
\end{gathered}
$$

With this choice of Feynman rules, the diagram of Fig. 3(a) corresponds to a vacuum polarization of the form

$\Pi_{W W}\left(k^{2}\right)=\frac{\beta^{2}}{8 \pi^{2}} \frac{\bar{B}^{\prime 2}}{\bar{B}} \int_{0}^{1} \mathrm{~d} x \int_{0}^{\Lambda} \frac{\kappa^{3} \mathrm{~d} \kappa}{\left(\kappa^{2}+\Sigma^{2}\right)^{2}}\left[\frac{\kappa^{2}}{4}\left(2 k^{2}+\gamma^{2} M_{W}^{2}\right)+\left(x k^{2}+\gamma M_{W}^{2}\right)^{2}\right]$,

where $x$ is a Feynman parameter, and we have Wick rotated to Euclidean signature before replacing the Euclidean volume element by $2 \pi^{2} \kappa^{3} \mathrm{~d} \kappa$. The momentum scale $\Lambda$ is a sharp cutoff which regulates the maximum Euclidean momentum permitted to 
circulate in the loop, and therefore determines the size of the phase space of scalar states to which each $W^{ \pm}$couples $\dagger$ Finally, $\Sigma^{2}$ is an abbreviation for the quantity

$$
\Sigma^{2} \equiv x(1-x) k^{2}+(1-x) M_{W}^{2}+x M_{\chi}^{2} .
$$

In writing Eqs. (33)-(34) we have freely replaced $m_{W}^{2}$ by $M_{W}^{2}$, since the correction this induces is formally of higher order in the loop expansion.

The diagram of Fig. 3(b) gives a somewhat simpler contribution,

$$
\Pi_{W W}\left(k^{2}\right)=-\frac{\beta^{2}}{8 \pi^{2}} \frac{\bar{B}^{\prime 2}}{\bar{B}} \int_{0}^{1} \mathrm{~d} x \int_{0}^{\Lambda} \frac{\kappa^{3} \mathrm{~d} \kappa}{\kappa^{2}+M_{\chi}^{2}} \frac{\Omega}{2}\left(k^{2}+\epsilon M_{W}^{2}\right),
$$

where $\Omega$ is a dimensionless combination which measures the curvature of the coupling function $B$ in the vacuum,

$$
\Omega \equiv \frac{\bar{B}^{\prime \prime} \bar{B}}{\bar{B}^{\prime 2}}
$$

We also require the vacuum polarization for the $Z$ boson and the photon, $\gamma$. However, no further calculation is required since the relevant Feynman rules can be obtained from (29) -(30), and the necessary vacuum polarizations can likewise be obtained from Eqs. (33)-(35). Since the $\gamma$ and $Z$ are their own antiparticles, each vertex in (29)-(30) acquires a symmetry factor of $1 / 2$. To obtain the correct vacuum polarizations, one makes the replacement $M_{W} \mapsto M_{Z}$ in Eqs. (33)-(35) for the $Z$, and $M_{W} \mapsto 0$ for the photon.

Assembling these terms and carrying out the $\kappa$ integrals, it follows that the vacuum polarization for each species of boson satisfies

$$
\begin{aligned}
\Pi_{A A}\left(k^{2}\right)=\frac{\beta^{2}}{8 \pi^{2}} & \frac{\bar{B}^{\prime 2}}{\bar{B}} \int_{0}^{1} \mathrm{~d} x\left\{\frac{2 k^{2}+\gamma^{2} M_{A}^{2}}{4}\left[\Lambda^{2}+\frac{\Lambda^{2}}{2} \frac{\Lambda^{2}}{\Lambda^{2}+\Sigma^{2}}-\Sigma^{2} \ln \left(1+\frac{\Lambda^{2}}{\Sigma^{2}}\right)\right]\right. \\
+ & \left(x k^{2}+\gamma M_{A}^{2}\right)^{2}\left[-\frac{1}{2} \frac{\Lambda^{2}}{\Lambda^{2}+\Sigma^{2}}+\frac{1}{2} \ln \left(1+\frac{\Lambda^{2}}{\Sigma^{2}}\right)\right] \\
& \left.-\frac{\Omega}{2}\left(k^{2}+\epsilon M_{A}^{2}\right)\left[\frac{\Lambda^{2}}{2}-\frac{M_{\chi}^{2}}{2} \ln \left(1+\frac{\Lambda^{2}}{M_{\chi}^{2}}\right)\right]\right\}
\end{aligned}
$$

\subsection{Effective Lagrangians for the vacuum polarization}

Eq. (37) is a complicated expression from which it is difficult to extract the important qualitative features of the oblique corrections. To do better, one can analyze $\Pi_{A A}\left(k^{2}\right)$ in terms of an effective Lagrangian which would give rise to the same vacuum polarization.

$\dagger$ Power-law divergences in $\Lambda$, if they exist, are likely to violate gauge invariant although logarithmic divergences should be physically meaningful. Also, loop calculations in unitarity gauge are known to overestimate power law divergences in certain circumstances. These issues were addressed in Refs. [27, 28]. In the present case it will turn out that we require only the logarithmic terms. If any power-law divergences were present, however, then it would not be possible to interpret the result as a quantitative prediction. Instead-provided such powers were compatible with naïve dimensional analysis (which excludes the possibility of overestimation) and the gauge symmetries of the model-the correct interpretation would be that the calculation under discussion was sensitive to the details of UV physics. 
Collider constraints on interactions of dark energy with the Standard Model

A. Low energy, massive vector bosons. Consider first the limit $\left|q^{2}\right| \ll M_{W}^{2}$. For each species of massive vector boson $A$ one can make the expansions

$$
\frac{\Lambda^{2}}{\Lambda^{2}+\Sigma^{2}}=\frac{1}{\sigma^{2}}+\frac{1}{\sigma^{2}} \sum_{n=1}^{\infty}(-1)^{n}\left[\frac{x(1-x)}{\sigma^{2}} \frac{k^{2}}{\Lambda^{2}}\right]^{n}
$$

and

$$
\ln \left(1+\frac{\Lambda^{2}}{\Sigma^{2}}\right)=\ln \frac{\sigma^{2}}{\sigma^{2}-1}+\sum_{m=1}^{\infty} \sum_{n=1}^{\infty} \frac{(-1)^{m(n+1)+1}}{m \sigma^{2 m}}\left[\frac{x(1-x) k^{2}}{(1-x) M_{A}^{2}+x M_{\chi}^{2}}\right]^{m n}
$$

where we have defined $\sigma^{2}$ by the rule

$$
\sigma^{2} \equiv 1+(1-x) \frac{M_{A}^{2}}{\Lambda^{2}}+x \frac{M_{\chi}^{2}}{\Lambda^{2}} .
$$

In particular, $\sigma^{2} \approx 1$ whenever the scale of the cutoff, $\Lambda$, is much larger than the electroweak scale $M_{\mathrm{EW}} \sim M_{A}$. Eq. (仿) is an expansion in powers of $k^{2} / \Lambda^{2}$. In an effective field theory, these contributions would come from a tower of non-renormalizable operators suppressed by the cutoff scale, although one should remember that whenever these operators become important the bridge corrections discussed in Appendix B will also make a significant contribution. On the other hand, Eq. (39) amounts to an expansion in powers of $k^{2} / M_{A}^{2} \ddagger$ These contributions would come from nonrenormalizable operators suppressed only by the electroweak scale. As we increase the momentum which is transferred through the gauge boson propagator from zero, we expect to see corrections enter at the scale $\left|k^{2}\right| \sim M_{A}^{2}$, followed by another set of corrections at the cutoff.

Collecting these expressions one finds an expansion for $\Pi_{A A}\left(k^{2}\right)$, which yields

$$
\Pi_{A A}\left(k^{2}\right)=\frac{g^{2}}{M^{2}}\left[M_{A}^{2} \alpha_{0}+\alpha_{2} k^{2}+\alpha_{4} k^{4}+\mathrm{O}\left(\frac{k^{2}}{M_{\mathrm{EW}}^{2}}\right)\right], \quad\left(\left|k^{2}\right| \ll M_{\mathrm{EW}}^{2}\right)
$$

where $g$ is an effective dimensionless coupling constant defined by

$$
g^{2} \equiv \frac{1}{8 \pi^{2}} \frac{\bar{B}^{\prime 2}}{\bar{B}}
$$

the mass scale $M$ is $M \equiv \beta^{-1}$, as before, and the coefficients $\alpha_{i}$, for $i \in\{0,2,4\}$, satisfy

$\alpha_{0} \equiv \frac{\Lambda^{2}}{4}\left(\frac{\gamma^{2}}{2}-\Omega \epsilon\right)+\frac{\gamma^{2} M_{A}^{2}}{16}\left[6 \ln \frac{\Lambda^{2}}{M_{A}^{2}}-1+\mathrm{O}\left(\frac{M_{\chi}^{2}}{M_{\mathrm{EW}}^{2}}\right)\right]$,

$\alpha_{2} \equiv \frac{\Lambda^{2}}{4}(1-\Omega)+\frac{M_{A}^{2}}{144}\left[6[\gamma(12-\gamma)-6] \ln \frac{\Lambda^{2}}{M_{A}^{2}}+\gamma(36-5 \gamma)-18+\mathrm{O}\left(\frac{M_{\chi}^{2}}{M_{\mathrm{EW}}^{2}}\right)\right]$,

$\alpha_{4} \equiv \frac{1}{12} \ln \frac{\Lambda^{2}}{M_{A}^{2}}+\frac{5}{72}+\mathrm{O}\left(\frac{M_{\chi}^{2}}{M_{\mathrm{EW}}^{2}}\right)$

$\ddagger$ The series expansion in Eq. (39) can be integrated term-by-term in $x$, producing an expansion in powers of $k^{2} / M_{A}^{2}$ with coefficients which involve hypergeometric functions of $M_{\chi}^{2} / M_{A}^{2}$. When expanded in powers of this ratio it is possible that logarithms of $M_{\chi}^{2} / M_{A}^{2}$ are generated, although suppressed by a positive power of $M_{\chi}^{2} / M_{A}^{2}$. It follows that the scale at which this tower of non-renormalizable operators becomes significant genuinely is around the electroweak scale, $\left|k^{2}\right| \sim M_{A}^{2}$. 
We could equally well have obtained this vacuum polarization if we had started from an action of the form

$$
\begin{aligned}
S=\frac{1}{2} \int \mathrm{d}^{4} x[(1 & \left.-\frac{g^{2}}{M^{2}} \alpha_{2}\right) \varphi \partial^{2} \varphi-M_{A}^{2}\left(1-\frac{g^{2}}{M^{2}} \alpha_{0}\right) \varphi^{2}-\frac{g^{2}}{M^{2}} \alpha_{4} \varphi \partial^{4} \varphi \\
& \left.+ \text { corrections at } M_{\mathrm{EW}}^{2}\right],
\end{aligned}
$$

and calculated only to tree level, where $\varphi$ represents any polarization of the vector boson of species $A$, and the corrections at $M_{\mathrm{EW}}^{2}$ take the form of a tower of non-renormalizable terms suppressed by powers of $M_{\mathrm{EW}}$. Note the unsuppressed non-renormalizable term of the form $\varphi \partial^{4} \varphi$, which is symptomatic of the fact that our starting Lagrangian, Eq. (2), did not describe a renormalizable quantum field theory.

A good deal of information can be obtained from inspection of the effective action (46). The relevant operators are the kinetic term $\varphi \partial^{2} \varphi$ and the mass term $\varphi^{2}$, which both receive corrections quadratic in the cutoff $\Lambda$. The mass is prevented from receiving corrections which scale faster than $\Lambda$ because gauge invariance is restored when $M_{A} \rightarrow 0$, and in this limit the mass must not receive quantum corrections so that the Ward identity is preserved. Indeed, it follows from Eq. (41) that the $T$ parameter can be written

$$
\alpha T=\frac{g^{2}}{M^{2}}\left(\alpha_{0, Z}-\alpha_{0, W}\right),
$$

and therefore that all quadratic divergences cancel in this quantity. It is clear from Eq. (46) that this cancellation is a direct consequence of the restoration of gauge invariance in the limit $M_{Z}, M_{W} \rightarrow 0$.

B. Low energy, massless vector bosons. A similar procedure can be applied to find an effective Lagrangian for the photon self-energy in the low-energy limit. The vacuum polarization is obtained from Eq. (37) after the replacement $M_{W} \mapsto 0$, after which the expansions (38)-(39) continue to apply, with $\sigma^{2}$ substituted by the alternative combination $\tau^{2}$, which satisfies

$$
\tau^{2} \equiv 1+x \frac{M_{\chi}^{2}}{\Lambda^{2}}
$$

However, the roles of these non-renormalizable operators are subtly changed. Eq. (38) can still be interpreted as a tower of corrections at the cutoff (which we again caution will be accompanied by significant bridge contributions), but Eq. (39) now represents corrections at the scale of the dark energy mass, $\left|k^{2}\right| \sim M_{\chi}^{2}$. If we discard these corrections, it follows that the effective Lagrangian we obtain will be valid only in the limit $\left|k^{2}\right| \ll M_{\chi}^{2}$. Fortunately, for finite $M_{\chi}$ this is sufficient for the purpose of obtaining the oblique parameter $S$.

In this limit, one finds

$$
\Pi_{\gamma \gamma}\left(k^{2}\right)=\frac{g^{2}}{M^{2}}\left[\delta_{2} k^{2}+\delta_{4} k^{4}+\mathrm{O}\left(\frac{k^{2}}{M_{\chi}^{2}}\right)\right], \quad\left(\left|k^{2}\right| \ll M_{\chi}^{2}\right)
$$


where the coefficients $\delta_{2}$ and $\delta_{4}$ satisfy

$$
\begin{aligned}
\delta_{2} & \equiv \frac{\Lambda^{2}}{4}(1-\Omega)+\mathrm{O}\left(M_{\chi}^{2}\right), \\
\delta_{4} & \equiv-\frac{1}{6}+\mathrm{O}\left(\frac{M_{\chi}^{2}}{\Lambda^{2}}\right) .
\end{aligned}
$$

Within its range of validity, this expansion can be interpreted in terms of the effective Lagrangian (46). In particular, note that (as expected), no mass term is generated owing to gauge invariance.

C. Energies near the resonance, massive vector bosons. To obtain $S$, we require information about $\Pi_{A A}\left(k^{2}\right)$ in the region where it approaches the resonance at $k^{2}=-M_{A}^{2}$. This can be studied by setting $k^{2}=-M_{A}^{2}+q^{2}$, and making an expansion in powers of $q^{2} / M_{A}^{2}$. When expanded in this way, it is less straightforward to interpret $\Pi\left(k^{2}\right)$ as an effective Lagrangian. However, some of our understanding concerning the meaning of each term can be carried over.

Eqs. (38)-(39), giving expansions in terms of non-renormalizable operators, continue to apply with the replacement $\sigma^{2} \mapsto \hat{\sigma}^{2}$, where for each species $A$ of massive vector boson we have defined

$$
\hat{\sigma}^{2} \equiv 1+(1-x)^{2} \frac{M_{A}^{2}}{\Lambda^{2}}+x \frac{M_{\chi}^{2}}{\Lambda^{2}} .
$$

We find

$$
\Pi_{A A}\left(k^{2}\right)=\frac{g^{2}}{M^{2}}\left[M_{A}^{2} \hat{\alpha}_{0}+\hat{\alpha}_{2} q^{2}+\hat{\alpha}_{4} q^{2}+\mathrm{O}\left(\frac{q^{2}}{M_{\mathrm{EW}}^{2}}\right)\right], \quad\left(\left|k^{2}\right| \sim M_{A}^{2}\right)
$$

where the coefficients $\hat{\alpha}_{i}$, for $i \in\{0,2,4\}$, satisfy

$$
\begin{aligned}
& \hat{\alpha}_{0} \equiv \frac{\Lambda^{2}}{4}\left(\frac{\gamma^{2}}{2}-\Omega(\epsilon-1)-1\right) \\
&+\frac{M_{A}^{2}}{36}\left(3[\gamma(5 \gamma-6)+4] \ln \frac{\Lambda^{2}}{M_{A}^{2}}+4[\gamma(4 \gamma-9)+5]+\mathrm{O}\left(\frac{M_{\chi}^{2}}{M_{\mathrm{EW}}^{2}}\right)\right), \quad(54) \\
& \hat{\alpha}_{2} \equiv \frac{\Lambda^{2}}{4}(1-\Omega)+\frac{M_{A}^{2}}{72}\left(3[\gamma(11 \gamma-16)+4] \ln \frac{\Lambda^{2}}{M_{A}^{2}}+\gamma(67 \gamma-128)+59+\mathrm{O}\left(\frac{M_{\chi}^{2}}{M_{\mathrm{EW}}^{2}}\right)\right),(55) \\
& \hat{\alpha}_{4} \equiv \frac{1}{12} \ln \frac{\Lambda^{2}}{M_{A}^{2}}+\frac{11}{36}+\mathrm{O}\left(\frac{M_{\chi}^{2}}{M_{\mathrm{EW}}^{2}}\right) .
\end{aligned}
$$

It is now possible to give expressions for the remaining oblique parameters $S, V$ and $W$ in terms of these effective quantities

$$
\begin{aligned}
& \frac{\alpha S}{4 s_{\mathrm{W}}^{2} c_{\mathrm{W}}^{2}}=\frac{g^{2}}{M^{2}}\left(\alpha_{0, Z}-\hat{\alpha}_{0, Z}-\delta_{2}\right), \\
& \alpha V=\frac{g^{2}}{M^{2}}\left(\hat{\alpha}_{2, Z}+\hat{\alpha}_{0, Z}-\alpha_{0, Z}\right), \\
& \alpha W=\frac{g^{2}}{M^{2}}\left(\hat{\alpha}_{2, Z}+\hat{\alpha}_{0, W}-\alpha_{0, W}\right),
\end{aligned}
$$


where we have dropped contributions from the non-renormalizable operator $\varphi \partial^{4} \varphi$ since these never lead to quadratic divergences. It is now clear from inspection of Eqs. (57)(59) together with Eqs. (43)-(45), (50)-(51) and (55)-(56) that all quadratic divergences cancel in $S, T, U V$ and $W$.

\section{Discussion}

\subsection{When are quantum corrections screened?}

This cancellation is not an accident, but is partly a consequence of gauge invariance and partly depends on the structure of gauge boson-lepton couplings within the Standard Model.

The available phase space which sets the size of the loop correction is determined by the couplings $\left\{B, B_{H}\right\}$ and the mass of the boson, which is an infra-red effect. Most of the phase space volume will be concentrated near the ultra-violet region, in spherical shells of large Euclidean four-momentum. Coupling to these shells corresponds to a process where a propagating intermediate vector boson radiates into a hard chameleon and boson pair. From the point of view of this pair, the original vector boson behaves as if it were massless, and the effect of mass splittings between $W^{ \pm}, Z$ and $\gamma$ becomes irrelevant. Therefore, because gauge invariance requires that $W^{ \pm}, Z$ and $\gamma$ couple to the dark energy in the same way at zero mass, we expect no difference in the manner in which any of these gauge bosons radiate into momentum shells at Euclidean fourmomenta which are large compared with $M_{Z}$.

Assuming our choice of input parameters, this is sufficient to screen all $\mathrm{O}(1)$ effects in contact interactions of a single electroweak gauge boson with exactly two fermionswhich is the only type of interaction which occurs in the electroweak sector, excluding interactions with the Higgs. The input parameters were chosen to be the $Z$ mass, $M_{Z}$, together with the fine structure constant, $\alpha$, and the Fermi constant, $G_{F}$, which measure the strength of the electromagnetic and charged-current interactions at zero momentum, respectively. Operationally, both $\alpha$ and $G_{F}$ measure a combination of some dimensionless coupling constants and a propagator at zero momentum: for $\alpha$ this is the photon propagator, whereas $G_{F}$ measures the $W$ propagator. The oblique corrections can be of two kinds. Firstly, for processes involving a $Z$ particle, the strength of the neutral-current coupling is not measured by $G_{F}$ but can be obtained from it by a shift measured at zero momentum. This is the purpose of the $T$ parameter. Secondly, a wavefunction renormalization of gauge boson lines may be necessary, which depends on properties of the propagator near Euclidean momentum of order $M_{Z}$. The difference between the wavefunction renormalization of the $Z$ and $W$ propagators evaluated at zero momentum and at momenta near $M_{Z}$ is measured by $V$ and $W$, respectively. Finally, $S$ compares the zero-momentum $Z$ and $\gamma$ propagators and therefore plays the same role for the photon as $T$ does for the $Z$, while $U$ measures the difference between the $W^{ \pm}$ and $Z$ propagators at zero momentum. 


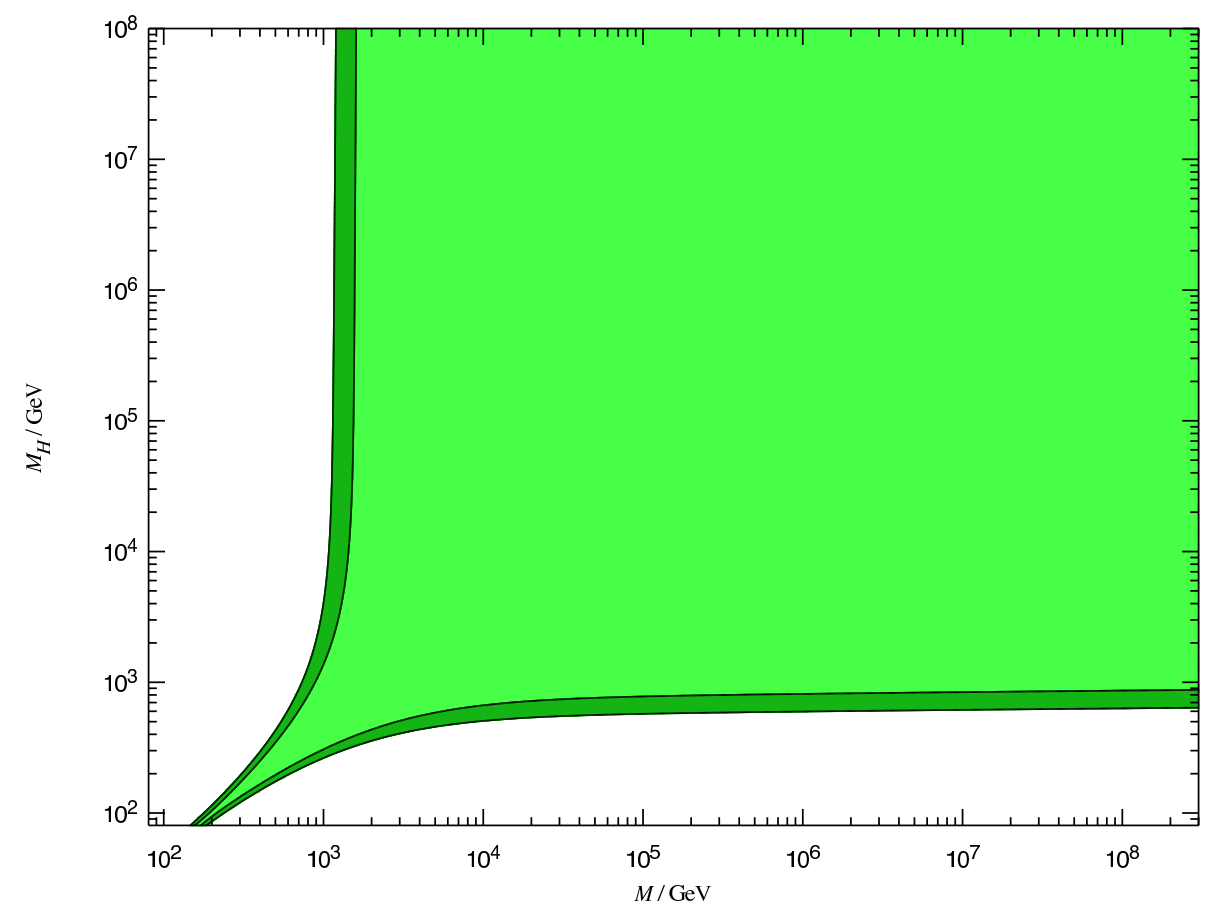

Figure 4. Current collider constraints on the coupling scales $M$ and $M_{H}$, associated with dark energy interactions with the electroweak gauge and Higgs sectors, respectively. The interior light green region is compatible with current precision electroweak data at $1 \sigma$, and extends indefinitely to large $M$ and $M_{H}$. Also shown is the $2 \sigma$ region in darker green.

All these shifts depend on a comparison of the phase space available to two different gauge bosons, or between the same gauge boson at different momenta. As we have seen, gauge invariance guarantees that the phase space available to all gauge bosons is the same at large Euclidean four-momentum, so differences can only arise from the interior shells of momentum space where the mass splitting between the electroweak gauge bosons can no longer be neglected. Differences in this region can not lead to $\mathrm{O}(1)$ effects if the mass scales $\left\{M, M_{H}\right\}$ associated with dark energy are much larger than the electroweak scale. It follows that large effects from radiative corrections are screened. However, this depends essentially on the fact that $\sqrt{\alpha}$ and $\sqrt{G_{F}}$ include one gauge boson line, and all other processes subsequently involve vertices which also include only a single ingoing or outgoing gauge boson.

The calculation of the oblique corrections in the previous sections was done only to one loop. In principle loop corrections of any order could contribute $\mathrm{O}(1)$ corrections but we expect that the screening of oblique scalar field corrections to the gauge boson propagators occurs at all orders.

For a dark energy species which selects its mass via a chameleon mechanism we depict the current collider constraints on the mass scales $M, M_{H}$ in Fig. 4. These can loosely be summarized as $M, M_{H} \gtrsim 1 \mathrm{TeV}$, which is stronger than the constraint which 


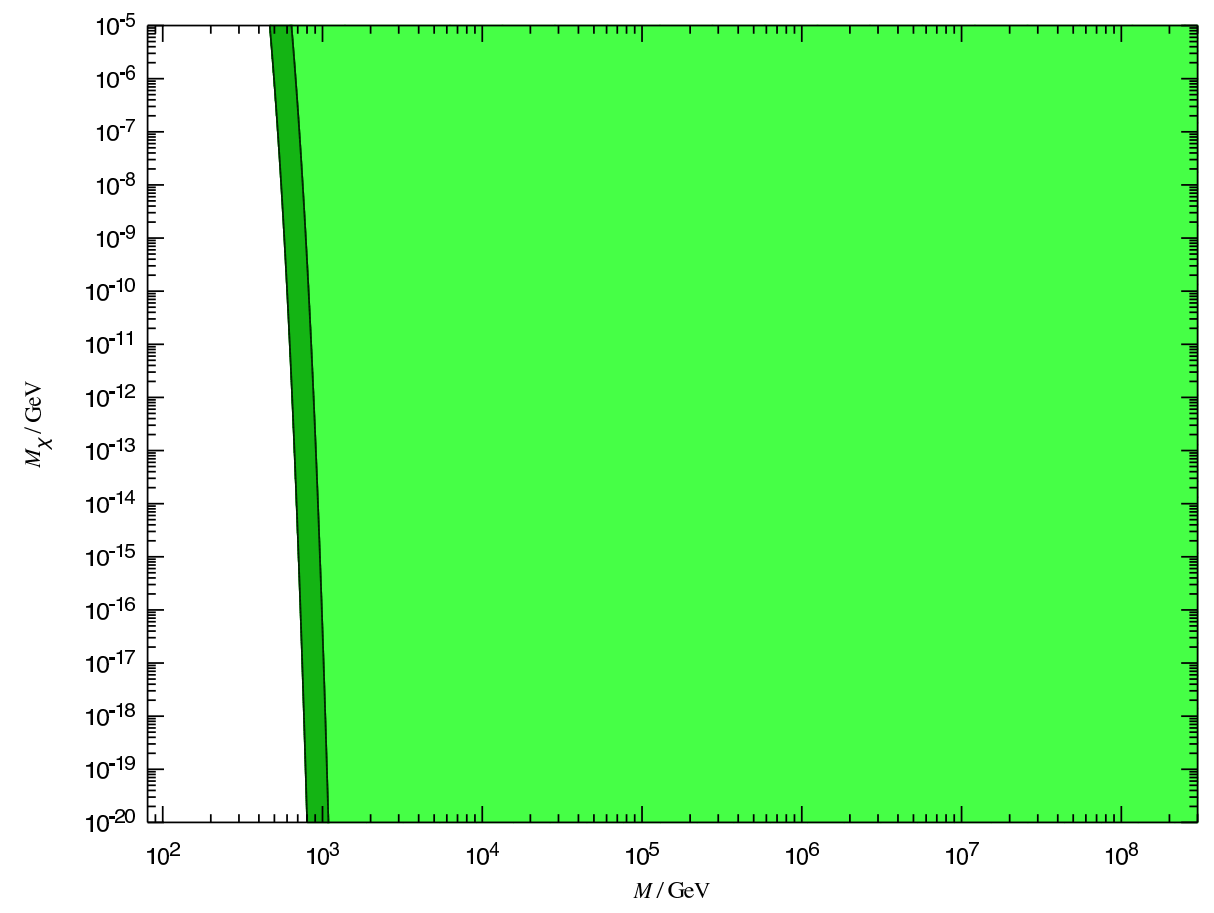

Figure 5. Current constraints on the mass, $M_{\chi}$, of a dark energy particle whose interaction with ordinary matter is characterized by a scale $M \gtrsim M_{\mathrm{EW}}$. The interior light green region is compatible with present data at $1 \sigma$, whereas the $2 \sigma$ region is shown in darker green. Compare with Fig. 8 of Ref. [42].

follows from the decay width of the $Z$ into visible particles. Clearly, neither constraint is competitive with present bounds from optical or axion-search observations [12, 43]. In Fig. 5 we show the same constraints as a function of the dark energy mass, $M_{\chi}$, and its interaction scale $M$, without assuming that $M_{\chi}$ is determined by some chameleon-type mechanism.

\subsection{Future prospects}

Any future linear collider will measure electroweak precision observables with remarkable accuracy [44], but if weak couplings imply it cannot produce dark energy particles directly then the most important discovery mode will come from sensitivity to radiative corrections at high energy. For any electroweak processes sensitive to the diverging phase space of dark energy states at large Euclidean four-momentum, the discovery reach of the ILC would not be limited by the smallness of the coupling unless new physics operating at lower energies could quench the contribution of dark energy loops. An example of such new physics could be the appearance of a chameleon superpartner at some energy $M_{\mathrm{SUSY}}$, if $M_{\mathrm{SUSY}} \ll M$, where $M$ is the characteristic mass scale with which dark energy couples to the gauge sector. On the other hand, if dark energy radiative corrections are screened, then contributions to electroweak precision observables fall with the mass 


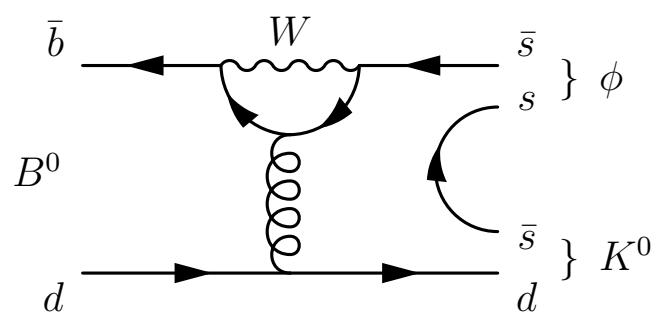

Figure 6. Penguin diagram contribution to the decay $B^{0} \rightarrow K^{0} \phi$.

scale of the coupling like $\left(M_{\mathrm{EW}} / M\right)^{2} \ln M^{2} / M_{\mathrm{EW}}^{2}$. The most stringent constraint on $M$ currently derives from the polarization of light from astrophysical sources, which was studied in Refs. [15, 45] and leads to the lower limit $M \gtrsim 10^{9} \mathrm{GeV}$. It is unlikely that such small corrections could ever be observed at the ILC.

Since detection of electroweakly interacting dark energy at $e^{+} e^{-}$colliders will be challenging, it is natural to consider what can be achieved at hadron-hadron colliders such as the LHC or the Tevatron. Although $W^{ \pm} \mathrm{s}$ and $Z \mathrm{~s}$ are produced by such colliders, the problem of backgrounds and the difficulty of kinematical reconstruction of the final state at a hadron collider mean that constraints from pure electroweak processes are likely to be inferior to those from a future linear collider. However, hadron colliders are sensitive to other channels in which new physics can appear. One particularly interesting window on new physics may be provided by rare decays of $B$ mesons, which are bound states of a bottom quark $b$ with some other quark $q$ in the combinations $b \bar{q}$ or $\bar{b} q$. Such mesons can decay via flavour-changing neutral currents which are forbidden at tree-level, but give rise to decays such as $B^{0} \rightarrow K^{0} \phi$ (where $B^{0}$ is the neutral $B$ meson composed of an anti-bottom/down pair $\bar{b} d$ ) when loop diagrams such as the so-called "penguin" of Fig. [6 are included. Rare processes of this type give a comparatively clean signal of new physics. Unfortunately, it does not appear likely that conformally coupled dark energy of the kind studied in this paper could manifest itself in this way. At large Euclidean fourmomentum, where the internal $W^{ \pm}$line in Fig. 6 could be expected to receive sizeable dark energy modifications, the quarks to which it couples are effectively massless and the loop is flavour-independent. When summed over all quarks which can circulate in the loop, the unitarity of the Cabbibo-Kobayashi-Maskawa matrix implies that this dominant flavour-independent contribution suffers an exact cancellation: this is the socalled Glashow-Iliopoulos-Maiani mechanism. We can estimate that this mechanism allows any dark energy contribution, coupling at a scale $M$, to contribute at most at relative order $m_{t}^{2} / M^{2}$, where $m_{t} \approx 175 \mathrm{GeV}$ is the top mass.

Is there any way to avoid the screening of large radiative corrections? This can only be done if at least one coupling constant measured in a low-energy interaction can appear in a different context in some other process. Remarkably, the Standard Model does allow for this possibility. If we assume a minimal Higgs sector, there are threeand four-body interactions of the massive electroweak gauge bosons with physical Higgs 
quanta which are described by the action

$S=-\int \mathrm{d}^{4} x\left(2^{1 / 4} \sqrt{G_{F}} H+G_{F} \frac{H^{2}}{\sqrt{2}}\right)\left(2 M_{W}^{2} W^{+a} W_{a}^{-}+M_{Z}^{2} Z^{a} Z_{a}\right)$,

where $H$ is the physical Higgs field. This must itself be subject to oblique corrections which only involve the coupling $B_{H}$. There is no reason to expect that the shifts necessary to bring $G_{F}$ and the $H$ and gauge boson lines to finite momenta will be independent of the ultra-violet region of momentum space. However, these large effects are undetectable until the interaction of the Higgs with at least one of the massive gauge bosons becomes accessible to experiment. Even when this is possible, the details will depend sensitively on the mechanism of electroweak symmetry breaking chosen by Nature. For this reason we defer investigation of such processes, although we note that in the case of a minimal $S U(2)$ doublet it is possible to verify that one could perhaps expect an $\mathrm{O}(1)$ modification of the Higgs production rate via weak boson fusion at the LHC.

The insensitivity of electroweak collider experiments to weakly coupled dark energy is frustrating given the inability of cosmological observations to place bounds on this region of parameter space. Although the search for astrophysical constraints has been fruitful [10, 15], it is difficult to imagine any astrophysical processes which would be sensitive to energy densities of order $\left(10^{12} \mathrm{GeV}\right)^{4}$ or above which were attained only during the very early universe. For example, one might have imagined that small perturbations imprinted in the dark energy scalar during an epoch of primordial inflation would lead to interesting effects in the late universe. Unfortunately, it appears that dark energy scalars of chameleon-type generically roll rapidly to their potential minima during the first few e-folds of inflation, where they remain for the duration of the accelerating era [5]. In the minimum, the dark energy field is heavy and is not excited by the inflationary expansion. For this reason, it does not function as an isocurvature field and cannot source evolution of the curvature perturbation, which might have led to interesting constraints from the spectral index or non-gaussianity. Moreover, the curvature perturbation is screened from possible non-perturbative effects because the chameleon vacuum expectation value is fixed [46]. On the other hand, if the chameleon vev shifted appreciably after inflation, it could potentially amplify the steep blue spectrum of perturbations imprinted on the $U(1)$ hypercharge field. If this amplification were too dramatic, it would lead to an unacceptable collapse of hypercharge fluctuations into primordial black holes at the end of inflation, in conflict with observation [47]. However, in practice the chameleon vev does not change sufficiently for this to provide an interesting constraint.

\section{Conclusions}

In this paper, we have studied the prospects for collider physics to detect a scalar dark energy species which couples conformally to Standard Model states which are charged under the electroweak gauge group $S U(2) \times U(1)$. This is particularly interesting for 
proposals incorporating a chameleon-type mechanism, in which the dark energy field may evade stringent bounds on the presence of light scalar bosons by dynamically adjusting its mass to be large in regions of high average density. Any such theory of dark energy must certainly couple to the Standard Model, although it is not mandatory that the dark energy scalar couples to electroweak states. However, if such couplings are present, then in view of the theoretical and experimental cleanliness of electroweak physics in comparison with hadron processes, one might expect that they would provide the most promising means of detection.

In the minimal Standard Model with Higgs sector consisting of only a single $S U(2)$ doublet, this expectation is wrong. Although coupling to a dark energy scalar in principle allows for fractional shifts of $\mathrm{O}(1)$ in precision electroweak observables, we have shown that in practice such large corrections are "screened," in direct analogy with the screening theorem which prevents similar corrections from heavy Higgs states. Screening occurs because a majority of the dark energy corrections are absorbed in the input parameters $\left\{\alpha, G_{F}, M_{Z}\right\}$, with only small splittings between the remaining observables which arise from the infra-red region of momentum space. The structure of the Standard Model also plays an important role, since all relevant vertices involve precisely two fermions and a single gauge boson. On the other hand, if it is possible to observe Higgs processes at the LHC then we would expect $\mathrm{O}(1)$ corrections to the Higgs production cross-section via weak-boson fusion.

Although we have carried our explicit calculations only to one loop, we expect that screening of oblique corrections persists to all orders, any of which could contribute $\mathrm{O}(1)$ effects as a matter of principle. This is important in establishing the consistency of dark energy theories with existing collider experiments, but also implies that the dark energy discovery potential of future $e^{+} e^{-}$colliders such as the proposed International Linear Collider may be comparatively limited, unless the Higgs can be detected.

One might also attempt to probe dark energy couplings via hadron processes, for which a promising observable might be the so-called flavour-changing neutral current which mediate rare decays of $B$ mesons. Unfortunately, for such reactions the unitarity of the Cabbibo-Kobayashi-Maskawa matrix plays a role similar to that of gauge invariance in quenching the contribution from shells of phase space at large Euclidean four-momentum, where a significant effect could be expected. Other non-electroweak effects such as bremsstrahlung also offer an apparently limited discovery potential.

\section{Acknowledgments}

It is a pleasure to thank Ben Allanach, Jeff Murugan and Malcolm Perry for helpful discussions. CB is supported by the German Science Foundation (DFG) under the Collaborative Research Centre (SFB) 676. ACD and DS are supported by STFC. AW is supported by the Cambridge Centre for Theoretical Cosmology. 


\section{Appendix A. Dark energy corrections to the $\mathrm{Z}$ width}

In this Appendix we discuss the possibility of enhancements to the observed decay width of the neutral $Z$ boson. Such enhancements arise via the process $Z \rightarrow \chi Z^{*} \rightarrow \chi \psi \bar{\psi}$ through which a propagating $Z$ emits a dark energy particle $\chi$ and passes off-shell. Eventually the off-shell $Z$ decays to a fermion-antifermion pair $Z^{*} \rightarrow \psi \bar{\psi}$, but if the weakly-interacting $\chi$ escapes the detector unseen then this reaction is indistinguishable from the direct decay $Z \rightarrow \psi \bar{\psi}$.

\section{Appendix A.1. Direct decay}

Let us first recapitulate the textbook calculation of direct decay [48]. This will allow us to express the enhancement from dark energy emission as a fraction of the pure Standard Model rate. We suppose that a $Z$ particle decays into a fermion species whose quanta are created and destroyed by operators associated with the Dirac fields $\psi$ and $\bar{\psi}$, according to an interaction Lagrangian of the form

$$
\int \mathrm{d}^{4} x \bar{\psi} \gamma^{a} Z_{a}\left(g_{L} L+g_{R} R\right) \psi
$$

where the $\gamma^{a}$ are matrices obeying the Dirac algebra $\left\{\gamma^{a}, \gamma^{b}\right\}=2 \eta^{a b}, g_{L}$ and $g_{R}$ are arbitrary left- (respectively, right-) handed couplings, and $L$ and $R$ are projections onto the left- (respectively, right-) chirality halves of a spinor in Dirac's representation,

$$
L \equiv \frac{1+\gamma_{5}}{2} \quad \text { and } \quad R \equiv \frac{1-\gamma_{5}}{2} .
$$

We use $\gamma_{5} \equiv-\mathrm{i} \gamma^{0} \gamma^{1} \gamma^{2} \gamma^{3}$, which has unit square $\gamma_{5}^{2}=1$ and commutes with all other $\gamma$-matrices. The projection operators $L$ and $R$ obey $L^{2}=L$ and $R^{2}=R$, together with the orthogonality relation $L R=R L=0$. We will also use the parity transformation operator $\beta=\mathrm{i} \gamma^{0}$, obeying $\beta^{2}=1$, in terms of which $\beta\left(\gamma^{a}\right)^{\dagger} \beta=-\gamma^{a}$ and $\beta \gamma_{5} \beta=-\gamma_{5}$.

Unpolarized decay proceeds according to the diagram of Fig. 1(a). To obtain the overall rate, one averages over the three polarizations of a massive spin-1 particle and sums over the two spin states of each outgoing fermion. The differential decay rate per unit volume of phase space, $\mathrm{d} v$, available to the final state $\psi \bar{\psi}$ pair corresponds to

$$
\frac{\mathrm{d} \Gamma}{\mathrm{d} v}=2 \pi \delta\left(\sum k\right) \sum_{\substack{\text { outgoing } \\ \text { spins }}} \frac{1}{3} \sum_{\substack{\text { ingoing } \\ \text { polarizations }}}\left|M_{\psi \bar{\psi}}\right|^{2}
$$

where $\sum k$ schematically denotes the sum of all ingoing momenta minus all outgoing momenta. The Feynman amplitude $M_{\psi \bar{\psi}}$ depends on the polarization of the initial $Z$, labelled $s$, and its 3 -momentum $\mathbf{p}$, together with the spins of the final-state fermions, labelled $\sigma_{1,2}$, and their 3 -momenta $\mathbf{t}_{1,2}$. It is defined by

$$
\left[M_{\psi \bar{\psi}}\right]^{s, \sigma_{1} \sigma_{2}} \equiv-\frac{1}{(2 \pi)^{3 / 2}}\left[\bar{u}^{\sigma_{1}}\left(\mathbf{t}_{1}\right) \gamma^{a}\left(g_{L} L+g_{R} R\right) v^{\sigma_{2}}\left(\mathbf{t}_{2}\right)\right] \frac{e_{a}^{s}(\mathbf{p})}{\sqrt{2 E_{Z}}} .
$$


After performing the spin and polarization sums, yielding a trace over Dirac indices, this corresponds to a differential decay rate which can be written

$$
\begin{aligned}
\mathrm{d} \Gamma=(2 \pi)^{4} \delta(p & \left.-t_{1}-t_{2}\right) \frac{\mathrm{d}^{3} t_{1}}{(2 \pi)^{3} 2 E_{1}} \frac{\mathrm{d}^{3} t_{2}}{(2 \pi)^{3} 2 E_{2}} \frac{1}{6 E_{Z}} \\
& \times\left\{12 g_{L} g_{R} M_{\psi}^{2}+\left(g_{L}^{2}+g_{R}^{2}\right)\left(4 \frac{\left(p \cdot t_{1}\right)\left(p \cdot t_{2}\right)}{M_{Z}^{2}}-2 t_{1} \cdot t_{2}\right)\right\}
\end{aligned}
$$

where $t_{1,2}=\left(E_{1,2}, \mathbf{t}_{1,2}\right)$ and $p=\left(E_{Z}, \mathbf{p}\right)$ are 4-momenta corresponding to the out-and in-going particles, respectively, and an infix dot '.' denotes contraction in the Minkowski metric. All external particles are taken to be on-shell, with 4-momentum conservation enforced by $\delta\left(p-t_{1}-t_{2}\right)$, and the outgoing fermions each have mass $M_{\psi}$.

Conservation of 3 -momentum is sufficient to determine one of the outgoing momenta. Moreover, performing the calculation in the $Z$ rest frame, symmetry requires that the outgoing fermions have equal energies $E_{1,2}=M_{Z} / 2$. In consequence, we conclude that the total rate of emission into a solid angle $\mathrm{d} \Omega$ can be written

$$
\frac{\mathrm{d} \Gamma}{\mathrm{d} \Omega}=\frac{M_{Z}}{96 \pi^{2}} \sqrt{1-4 \frac{M_{\psi}^{2}}{M_{Z}^{2}}}\left\{6 g_{L} g_{R} \frac{M_{\psi}^{2}}{M_{Z}^{2}}+\left(g_{L}^{2}+g_{R}^{2}\right)\left(1-\frac{M_{\psi}^{2}}{M_{Z}^{2}}\right)\right\} .
$$

\section{Appendix A.2. Decay accompanied by dark energy emission}

Now we return to the more complicated process where the decaying $Z$ is first pushed off-shell via emission of a single $\chi$ particle and subsequently decays into the observed fermion pair. This corresponds to the process of Fig. 1(b). The $Z Z \chi$ interaction vertex is determined by (29), modified as discussed below Eq. (36) to obtain the coupling to the $Z$ boson.

As above we label the decaying $Z$ with momentum $\mathbf{p}$ and energy $E_{Z}$, and the outgoing fermions with momenta $\mathbf{t}_{1,2}$ and energies $E_{1,2}$. The outgoing $\chi$ particle is taken to have momentum $\mathbf{q}$ and energy $E_{\chi}$. The total decay rate per unit of phase space available to the particles in the final state is given by a formula equivalent to Eq. (A.3), with the Feynman amplitude $M_{\psi \bar{\psi}}$ replaced by a more complicated quantity $M_{\chi \psi \bar{\psi}}$ which satisfies

$$
\begin{aligned}
{\left[M_{\chi \psi \bar{\psi}}\right]^{s, \sigma_{1} \sigma_{2}} \equiv } & -\frac{1}{(2 \pi)^{3}} \frac{\bar{B}^{\prime} M^{-1}}{r^{2}+M_{Z}^{2}}\left[\eta^{a b}\left(-p \cdot r-\gamma M_{Z}^{2}\right)+p^{b} r^{a}\right]\left(\eta_{b c}+\frac{r_{b} r_{c}}{M_{Z}^{2}}\right) \\
& \times\left[\bar{u}\left(\mathbf{t}_{1}\right) \gamma^{c} G v\left(\mathbf{t}_{2}\right)\right] \frac{e_{a}^{s}(\mathbf{p})}{\sqrt{2 E_{Z}}} \frac{1}{\sqrt{2 E_{\chi}}} .
\end{aligned}
$$

In order to avoid confusion with the parity inversion operator $\beta \equiv \mathrm{i} \gamma^{0}$ we have chosen the chameleon coupling scale as $M$, which elsewhere in this paper has been been synonymous with the coupling $\beta=M^{-1}$. The off-shell interior $Z$ carries 4-momentum $r=p-q$, and $G$ is the 'coupling matrix,'

$$
G_{\alpha \beta} \equiv\left[g_{L} L+g_{R} R\right]_{\alpha \beta}
$$


where $\{\alpha, \beta, \ldots\}$ label spinor indices. Summing over final-state spins and averaging over all three initial-state polarizations, we find

$\mathrm{d} \Gamma=(2 \pi)^{4} \delta\left(p-q-t_{1}-t_{2}\right) \frac{\bar{B}^{\prime 2} M^{-2}}{\left(r^{2}+M_{Z}^{2}\right)^{2}} \frac{\mathrm{d}^{3} q}{(2 \pi)^{3} 2 E_{\chi}} \frac{\mathrm{d}^{3} t_{1}}{(2 \pi)^{3} 2 E_{1}} \frac{\mathrm{d}^{3} t_{2}}{(2 \pi)^{3} 2 E_{2}} \frac{1}{6 E_{Z}} \mathcal{M}_{\chi \psi \bar{\psi}}^{\prime}$,

where $\mathcal{M}_{\chi \psi \bar{\psi}}^{\prime}$ satisfies

$\mathcal{M}_{\chi \psi \bar{\psi}}^{\prime} \equiv P_{c}^{a} P_{f}^{d}\left(\eta_{a d}+\frac{p_{a} p_{d}}{M_{Z}^{2}}\right) \operatorname{tr}\left\{\gamma^{c} G(-1)\left(\mathrm{i} t_{2}+M_{\psi}\right) \beta(G)^{\dagger}\left(\gamma^{f}\right)^{\dagger} \beta\left(-\mathrm{i} t_{1}+M_{\psi}\right)\right\}$

We are adopting the usual Feynman convention in which $\not \geq \gamma^{a} Z_{a}$ for any 4 -vector $Z$; and $P^{a}{ }_{c}$ is defined by

$$
P_{c}^{a} \equiv\left\{\eta^{a b}\left(-p \cdot r-\gamma M_{Z}^{2}\right)+p^{b} r^{a}\right\}\left(\eta_{b c}+\frac{r_{b} r_{c}}{M_{Z}^{2}}\right) .
$$

The trace over Dirac indices can be evaluated by standard methods. It yields

trace $=\eta^{c f}\left\{4 g_{L} g_{R} M_{\psi}^{2}-2\left(g_{L}^{2}+g_{R}^{2}\right) t_{1} \cdot t_{2}\right\}+2\left(g_{L}^{2}+g_{R}^{2}\right)\left(t_{2}^{c} t_{1}^{f}+t_{2}^{f} t_{1}^{c}\right)$,

plus a term antisymmetric under the exchange $c \leftrightarrow f$, which we omit because it disappears after insertion in Eq. (A.10). This trace depends only on the final $Z \psi \bar{\psi}$ vertex and is common between the direct and accompanied decays. Nevertheless, it will not cancel in a ratio between the two, because it depends non-trivially on the Lorentz index structure by which it couples to the rest of the diagram. This structure receives significant modifications when the $Z$ decay is accompanied by dark energy emission.

To proceed, we must contract Lorentz indices. We find

$$
\begin{aligned}
\left(\eta_{a d}+\frac{p_{a} p_{d}}{M_{Z}^{2}}\right) P_{c}^{a} P_{f}^{d} & =p_{c} p_{f}\left(r^{2}+\gamma^{2} M_{Z}^{2}\right)+\eta_{c f}\left(p \cdot r+\gamma M_{Z}^{2}\right)^{2} \\
+ & +r_{c} r_{f}\left[\left(\gamma-\frac{p \cdot r}{M_{Z}^{2}}\right)\left(1+\frac{r^{2}}{M_{Z}^{2}}\right)\left(p \cdot r+\gamma M_{Z}^{2}\right)\right. \\
& \left.\left.\quad+\frac{1}{M_{Z}^{2}}\left(p \cdot r+\gamma M_{Z}^{2}\right)^{2}+\left(\frac{p \cdot r}{M_{Z}^{2}}\right)^{2}\left(r^{2}+\gamma^{2} M\right) Z^{2}\right)\right] \\
+ & \left(r_{c} p_{f}+p_{c} r_{f}\right)\left[\frac{p \cdot r}{M_{Z}^{2}}\left(r^{2}+\gamma^{2} M_{Z}^{2}\right)-\left(1+\frac{r^{2}}{M_{Z}^{2}}\right)\left(p \cdot r+\gamma M_{Z}^{2}\right)\right]
\end{aligned}
$$

This can be contracted with Eq. (A.12) for the Dirac trace, yielding a final expression for $\mathcal{M}_{\chi \psi \bar{\psi}}^{\prime}$. For convenience of expression, let us write $\mathcal{M}_{\chi \psi \bar{\psi}}^{\prime}=\mathcal{A}+\mathcal{B}$. We find

$$
\begin{gathered}
\frac{\mathcal{A}}{4 M_{\psi}^{2} g_{L} g_{R}-2\left(g_{L}^{2}+g_{R}^{2}\right) t_{1} \cdot t_{2}} \equiv-M_{Z}^{2}\left(r^{2}+\gamma^{2} M_{Z}^{2}\right)+4\left(p \cdot r+\gamma M_{Z}^{2}\right)^{2} \\
+r^{2}\left[\left(\gamma-\frac{p \cdot r}{M_{Z}^{2}}\right)\left(1+\frac{r^{2}}{M_{Z}^{2}}\right)\left(p \cdot r+\gamma M_{Z}^{2}\right)\right. \\
\left.+\frac{\left(p \cdot r+\gamma M_{Z}^{2}\right)^{2}}{M_{Z}^{2}}+\left(\frac{p \cdot r}{M_{Z}^{2}}\right)^{2}\left(r^{2}+\gamma^{2} M_{Z}^{2}\right)\right] \\
+2(p \cdot r)\left[\frac{p \cdot r}{M_{Z}^{2}}\left(r^{2}+\gamma^{2} M_{Z}^{2}\right)-\left(1+\frac{r^{2}}{M_{Z}^{2}}\right)\left(p \cdot r+\gamma M_{Z}^{2}\right)\right]
\end{gathered}
$$


and

$$
\begin{aligned}
\frac{\mathcal{B}}{2\left(g_{L}^{2}+g_{R}^{2}\right)} \equiv 2\left(p \cdot t_{1}\right)\left(p \cdot t_{2}\right)\left(r^{2}+\gamma^{2} M_{Z}^{2}\right)+2\left(t_{1} \cdot t_{2}\right)\left(p \cdot r+\gamma M_{Z}^{2}\right)^{2} \\
+2\left(r \cdot t_{1}\right)\left(r \cdot t_{2}\right)\left[\left(\gamma-\frac{p \cdot r}{M_{Z}^{2}}\right)\left(1+\frac{r^{2}}{M_{Z}^{2}}\right)\left(p \cdot r+\gamma M_{Z}^{2}\right)\right. \\
\left.\quad+\frac{\left(p \cdot r+\gamma M_{Z}^{2}\right)^{2}}{M_{Z}^{2}}+\left(\frac{p \cdot r}{M_{Z}^{2}}\right)^{2}\left(r^{2}+\gamma^{2} M_{Z}^{2}\right)\right] \\
+2\left[\left(p \cdot t_{1}\right)\left(r \cdot t_{2}\right)+\left(p \cdot t_{2}\right)\left(r \cdot t_{1}\right)\right] \\
\quad \times\left[\frac{p \cdot r}{M_{Z}^{2}}\left(r^{2}+\gamma^{2} M_{Z}^{2}\right)-\left(1+\frac{r^{2}}{M_{Z}^{2}}\right)\left(p \cdot r+\gamma M_{Z}^{2}\right)\right]
\end{aligned}
$$

Kinematics. As before, 3-momentum conservation is sufficient to determine the momentum of one outgoing particle, which we choose to be $\mathbf{t}_{2}$ without loss of generality. Energy conservation determines one further scalar coordinate on phase space, which we choose to be $E_{1}$. The undetermined part of the 3-body phase space is parametrized by the outgoing dark energy momentum $\mathbf{q}$ and a pair of polar and azimuthal angles $(\theta, \phi)$ which specify the orientation of $\mathbf{t}_{1}$ relative to $\mathbf{q}$.

Let us obtain $E_{1}$ as a function of the unconstrained parameters. We work in the $Z$ rest frame. Energy conservation requires $E_{\chi}+E_{1}+E_{2}=M_{Z}$, and 3-momentum conservation fixes $\mathbf{t}_{2}=-\mathbf{t}_{1}-\mathbf{q}$. Therefore we conclude that $E_{1}$ must solve the implicit equation

$$
E_{2}^{2}=E_{1}^{2}+E_{\chi}^{2}-M_{\chi}^{2}+2 \sqrt{E_{\chi}^{2}-M_{\chi}^{2}} \sqrt{E_{1}^{2}-M_{\psi}^{2}} \cos \theta .
$$

To obtain an explicit equation, Eq. (A.16) can be squared and the resulting relation simplified. However, in doing so we introduce an extra solution for which Eq. (A.16) holds only after the exchange $\cos \theta \mapsto-\cos \theta$. The solution is spurious and should be eliminated. In practice we will find that the two possible solutions exchange roles at $\theta=\pi / 2$, but that the matching is smooth. Following this procedure, the possible solutions must solve the quadratic equation

$$
\begin{gathered}
E_{1}^{2}\left[4 \cos ^{2} \theta\left(E_{\chi}^{2}-M_{\chi}^{2}\right)-4\left(M_{Z}-E_{\chi}\right)^{2}\right]+4 E_{1}\left(M_{Z}-E_{\chi}\right)\left(M_{Z}^{2}+M_{\chi}^{2}-2 M_{Z} E_{\chi}\right) \\
=\left(M_{Z}^{2}+M_{\chi}^{2}-2 M_{Z} E_{\chi}\right)^{2}+4 M_{\psi}^{2} \cos ^{2} \theta\left(E_{\chi}^{2}-M_{\chi}^{2}\right) .
\end{gathered}
$$

This can be achieved by substituting the correct solution of Eq. (A.17) in Eqs. (A.9)(A.10). However, one must also account for a Jacobian factor associated with transformation of the $\delta$-function enforcing energy conservation. To obtain this, define

$$
\mathcal{E} \equiv E_{Z}-E_{\chi}-E_{1}-E_{2}\left(E_{\chi}, E_{1}, \theta\right),
$$

in terms of which energy conservation requires $\delta(\mathcal{E})$. We can now use a change of variables to find

$$
\delta(\mathcal{E})=\frac{\delta\left[E_{1}-E_{1}\left(E_{\chi}, \theta\right)\right]}{\left|\partial \mathcal{E} / \partial E_{1}\right|},
$$


where $E_{1}\left(E_{\chi}, \theta\right)$ is a solution of Eq. (A.17). The Jacobian $\partial \mathcal{E} / \partial E_{1}$ can be determined using Eq. (A.16), yielding

$$
\left|\frac{\partial \mathcal{E}}{\partial E_{1}}\right|=\left|1+E_{1} \frac{1+\left(E_{\chi}^{2}-M_{\chi}^{2}\right)^{1 / 2}\left(E_{1}^{2}-M_{\psi}^{2}\right)^{-1 / 2} \cos \theta}{E_{2}}\right|,
$$

where $E_{2}$ is to be determined by Eq. (A.16). In sum, the total unpolarized decay rate now satisfies

$$
\frac{\mathrm{d} \Gamma}{\mathrm{d} \Omega_{1}}=\frac{\bar{B}^{\prime 2} M^{-2}}{192 \pi^{2}(2 \pi)^{3}} \frac{\sqrt{E_{1}^{2}-M_{\psi}^{2}}}{\left(r^{2}+M_{Z}^{2}\right)^{2}} \frac{\mathrm{d}^{3} q}{E_{\chi} E_{2} M_{Z}}\left|\frac{\partial \mathcal{E}}{\partial E_{1}}\right|^{-1} \mathcal{M}_{\chi \psi \bar{\psi}}^{\prime},
$$

where $\mathrm{d} \Omega_{1}$ is the element of solid angle associated with $\mathbf{t}_{1}$.

To proceed it is convenient to introduce dimensionless small quantities $x$ and $y$, given in Eqs. (4) -(5), which determine $M_{\chi}$ and $M_{\psi}$ in terms of $M_{Z}$,

$$
M_{\psi}=\sqrt{x} M_{Z} \text { and } M_{\chi}=\sqrt{y} M_{Z} .
$$

Also, we can agree to measure energies in units of $M_{Z}$, introducing quantities $\hat{E}_{\chi}$ and $\hat{E}_{1,2}$ which satisfy Eq. (6) . Likewise, vectors such as $\mathbf{r}$ and $\mathbf{q}$ can be rescaled according to Eq. (7), giving dimensionless vectors $\hat{\mathbf{r}}$ and $\hat{\mathbf{q}}$. In terms of these dimensionless quantities, Eq. (A.6) giving the rate of direct decay reads

$$
\frac{\mathrm{d} \Gamma(Z \rightarrow \psi \bar{\psi})}{\mathrm{d} \Omega}=\frac{M_{Z}}{96 \pi^{2}} \mathcal{M}_{\psi \bar{\psi}}
$$

where $\mathcal{M}_{\psi \bar{\psi}}$ satisfies Eq. (11) with the fermion species $f$ taken to be $\psi$. The Jacobian $\left|\partial \mathcal{E} / \partial E_{1}\right|$ satisfies Eq. (10), and we will denote it $J$ in what follows. Moreover, $\mathcal{M}_{\chi \psi \bar{\psi}}^{\prime}$ has mass dimension $\left[M^{6}\right]$, so we can introduce an unprimed quantity $\mathcal{M}_{\chi \psi \bar{\psi}}$ which depends only on $x, y$, the hatted vectors and other dimensionless quantities, and is defined by

$$
\mathcal{M}_{\chi \psi \bar{\psi}}^{\prime} \equiv M_{Z}^{6} \mathcal{M}_{\chi \psi \bar{\psi}}
$$

After these replacements, the rate of unpolarized decay accompanied by dark energy emission satisfies

$$
\frac{\mathrm{d} \Gamma(Z \rightarrow \chi \psi \bar{\psi})}{\mathrm{d} \Omega_{1}}=\frac{\bar{B}^{\prime 2} M^{-2}}{192 \pi^{2}(2 \pi)^{3}} M_{Z}^{3} \mathrm{~d} \hat{E}_{\chi} \mathrm{d} \Omega_{\chi} \frac{\sqrt{\hat{E}_{1}^{2}-x^{2}} \sqrt{\hat{E}_{\chi}^{2}-y^{2}}}{J\left(1-\hat{E}_{\chi}-\hat{E}_{1}\right)\left(1+\hat{r}^{2}\right)^{2}} \mathcal{M}_{\chi \psi \bar{\psi}}
$$

Taking the ratio of Eqs. (A.25) and (A.23) we finally obtain our advertised relation, Eq. (3), which describes the enhancement due to dark energy emission as a fraction of the bare Standard Model rate. As in Eq. (12), it is convenient to aggregate that part of the enhancement in Eq. (A.25) which is independent of the chameleon coupling $M$ into a dimensionless integral $I_{\chi \psi \bar{\psi}}$. This will depend on the mass and couplings of the fermion species $\psi$, together with the mass of the dark energy scalar $\chi$. In particular,

$I_{\chi \psi \bar{\psi}}\left(M_{\psi}, M_{\chi}, g_{L}, g_{R}\right) \equiv \int \mathrm{d} \hat{E}_{\chi} \mathrm{d} \Omega_{\chi} \frac{\sqrt{\hat{E}_{1}^{2}-x^{2}} \sqrt{\hat{E}_{\chi}^{2}-y^{2}}}{J\left(1-\hat{E}_{\chi}-\hat{E}_{1}\right)\left(1+\hat{r}^{2}\right)^{2}} \frac{\mathcal{M}_{\chi \psi \bar{\psi}}}{\mathcal{M}_{\psi \bar{\psi}}}$.

We give representative values for $I_{\chi \psi \bar{\psi}}$ in Table A1. 


\begin{tabular}{c|ccccc}
\hline fermion species & massless neutrino & $10^{-3} \mathrm{eV}$ neutrino & $511 \mathrm{keV}$ electron & $5 \mathrm{GeV}$ electron & $40 \mathrm{GeV}$ electron \\
\hline$I_{\chi \psi \bar{\psi}}$ & 0.22 & 0.22 & 0.22 & 0.21 & 0.007 \\
\hline
\end{tabular}

Table A1. Enhancement factors for $Z$ decay accompanied by dark energy emission, to be interpreted in conjunction with Eqs. (12) and (A.26). As the fermion mass increases, the phase space available to any decay products diminishes until it is forbidden altogether at the kinematic threshold $M_{\psi}=M_{Z} / 2$. The enhancement for massless or light particles is very nearly independent of their identity.

\section{Appendix B. Bridges and daisies: dark-energy corrections to all orders}

Even in the effective field theory interpretation, where loops which are purely internal to the dark energy sector are ignored, Eq. (2) - together with the assumption that all matter fields couple conformally - entails a great many possible corrections to Standard Model processes. In this Appendix, we argue that to an acceptable approximation a majority of these corrections are subdominant; in this approximation, the leading dark energy effect comes from the one-loop oblique correction. This assumption played an essential role in determining the dark energy corrections in $\$ 3$,

A useful example to keep in mind is the case of the graviton, which also couples conformally to matter (and indeed all Standard Model states) with a universal coupling function, $\sqrt{\operatorname{det}\left(\eta_{a b}+h_{a b}\right)}$, where $\eta_{a b}$ is the background metric and $h_{a b}$ is the spin-2 graviton field. This non-linear coupling leads to a network of gravitational bridges, daisies and oblique corrections, quite analogous to Fig. 2, which also dress Standard Model processes. In the case of gravitons these have very little impact on reactions taking place at collider energies; in comparison, the structure of the dark energy interactions in Eq. (2) allow a small number of diagrams to make an $O(1)$ contribution. Nevertheless, many similarities exist between graviton and dark energy phenomenology.

Together with the simple daisy and bridge classes introduced in Fig. 2, one can contemplate more complicated corrections. Bridges can be chained together in arbitrary combinations, as shown in Fig. B1(a), or the component lines within a given bridge can themselves be joined together by other particles, as in Fig. B1(b). Alternatively, bridges can be nested within each other to create rainbows - see Fig. B1(c). In principle, a hierarchy of resummations (somewhat similar to the Balitsky hierarchy in QCD) is necessary to accommodate all these types of activity.

\section{Appendix B.1. Daisy diagrams}

Let us first consider the effect of daisies which dress bare Standard Model vertices. These are always momentum-independent and merely constitute a renormalization of whichever coupling constant sets the strength of the interaction at the vertex. For this reason they are relatively straightforward to deal with, and in the simplest situation we shall be able to resum their effect to all orders. If the daisies vary between different species of fermion, then the result would be an apparent species-dependent Fermi 

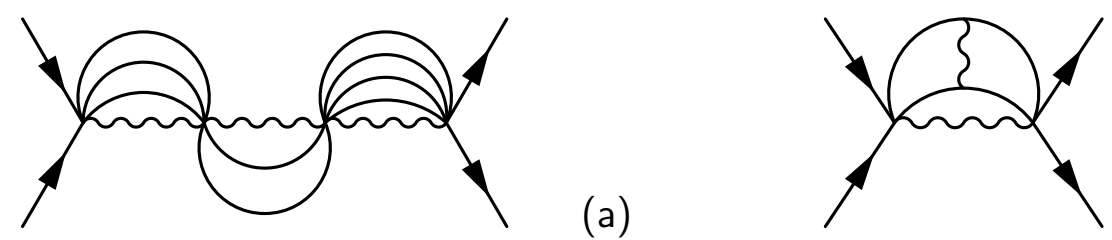

(b)

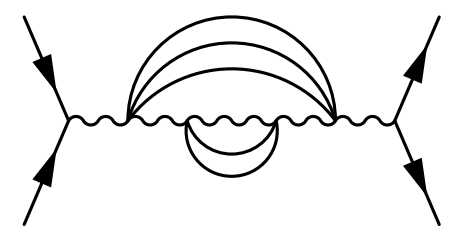

(c)

Figure B1. More complicated bridge-class diagrams. In addition to the simple bridge shown in Fig. 2(c), one can use high-order vertices between two gauge bosons and an arbitrary number of dark energy quanta to "chain" any number of bridges together, as shown in (a). In (b), the component lines of a particular bridge are themselves joined together by virtual quanta of other species. (These could include dark energy particles, because the loops formed by such "joined bridges" would not already be included in the effective dark energy sector.) In (c), bridge diagrams are nested within each other to form so-called rainbow diagrams.

constant, $G_{F}$. To prevent this occurring the fermion coupling function must be universal, which will be the case for conformal couplings. In what follows, we assume this to be the case.

Consider Eq. (2) and expand the coupling functions $B(x)$ and $B_{H}(x)$ according to

$$
\begin{aligned}
& B(\beta \chi) \equiv \sum_{n=0}^{\infty} \frac{1}{n !} \bar{B}_{n} \beta^{n}(\delta \chi)^{n} \\
& B_{H}(\beta \chi) \equiv \sum_{n=0}^{\infty} \frac{1}{n !} \bar{B}_{H, n} \beta_{H}^{n}(\delta \chi)^{n},
\end{aligned}
$$

where $\chi=\bar{\chi}+\delta \chi$, given that $\bar{\chi}$ is the expectation value of the dark energy scalar in the vacuum, and $\bar{B}_{n}\left(\bar{B}_{H, n}\right)$ are the Taylor coefficients of $B\left(B_{H}\right)$ evaluated in this vacuum. We assume that $\bar{B}_{0}=\bar{B}_{H, 0}=1$ and introduce a quantity $\gamma_{n}$, defined by

$$
\gamma_{n} \equiv \frac{\bar{B}_{H, n} \beta_{H}^{n}}{\bar{B}_{n} \beta^{n}} .
$$

In terms of $\bar{B}_{n}$ and $\gamma_{n}$, the $n$th order interaction vertex takes the form

$$
\begin{aligned}
S_{n}=\int \frac{\mathrm{d}^{4} k_{1}}{(2 \pi)^{4}} & \frac{\mathrm{d}^{4} k_{2}}{(2 \pi)^{4}} \frac{\mathrm{d}^{4} p_{1}}{(2 \pi)^{4}} \cdots \frac{\mathrm{d}^{4} p_{n}}{(2 \pi)^{4}}(2 \pi)^{4} \delta\left(k_{1}+k_{2}+\sum_{j} p_{j}\right) \\
& \frac{\bar{B}_{n} \beta^{n}}{n !} W_{a}^{+}\left(k_{1}\right) W_{b}^{-}\left(k_{2}\right) \delta \chi\left(p_{1}\right) \cdots \delta \chi\left(p_{n}\right)\left[\eta^{a b}\left(k_{1} \cdot k_{2}-\gamma_{n} M_{W}^{2}\right)-k_{1}^{b} k_{2}^{a}\right] .
\end{aligned}
$$

As an example, we will compute the simplest class of daisies which contribute to the interior of the $W^{ \pm}$propagator. The calculation of daisies which dress vertices with other species of fermion - or for the other gauge bosons - proceeds analogously. One finds that 
the vacuum polarization with momentum transfer $q$, which arises from the daisy with $n$ petals, can be written

$\Pi_{a b}^{n} \supseteq-\bar{B}_{2 n} \beta^{2 n} \frac{(n-1) ! !}{(2 n) !}\left\{\int \frac{2 \pi^{2} \kappa^{3} \mathrm{~d} \kappa}{(2 \pi)^{4}} \frac{1}{\kappa^{2}+M_{\chi}^{2}}\right\}^{n}\left[\eta_{a b}\left(q^{2}+\gamma_{n} M_{W}^{2}\right)-q^{a} q^{b}\right]$,

where $(n-1) ! ! \equiv(n-1)(n-3) \cdots 1$ is the so-called "double factorial." In the special case of an exponential coupling, where $\bar{B}_{n}=1$ for all $n$, and assuming that $\gamma_{n}$ can be replaced by a constant $\gamma$, then it follows that all orders of daisies can be resummed to give

$$
\begin{aligned}
& \Pi_{a b} \supseteq-\left[\eta_{a b}\left(q^{2}+\gamma M_{W}^{2}\right)-q_{a} q_{b}\right]\left\{-1+F\left(\begin{array}{c|c}
\cdot & (\beta \Lambda)^{4} \\
1 / 4,3 / 4 & \frac{M^{15} \pi^{4}}{2}
\end{array} 1-\frac{\Lambda^{2}}{\Lambda^{2}} \ln \frac{M_{\chi}^{2}}{M_{\chi}^{2}}\right)\right.
\end{aligned}
$$

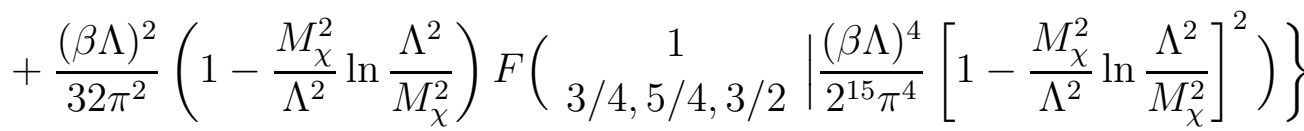

where $F(a ; b \mid z)$ is the generalized hypergeometric function. For $\Lambda \lesssim \beta^{-1}$ and $M_{\chi} \ll \Lambda$ this resummation is dominated by its one-loop term. There will be extra terms in addition to Eq. (B.6) which arise from interference between daisy diagrams and bridge diagrams (to be discussed in the next section). Although these may change the details of some numerical coefficients, they will not alter the momentum-independent character of the corrections.

Eqs. (B.5) - (B.6) exhibit the general features which will recur in all diagrams we consider in this Appendix. A diagram with $n$ dark energy lines can contribute at leading order in powers of $\beta \Lambda$, with this contribution coming from a region of phase space where all dark energy lines are carrying momenta of order $\Lambda$. This would seem to suggest that diagrams containing an arbitrary number of lines need to be accounted for in order to make reliable predictions. However, each line is also accompanied by a phase-space factor of $1 / 16 \pi^{2}$ (plus the combinatorical factor $\left.(n-1) ! ! /(2 n) !\right)$ which leads to suppression of high-loop terms, so that counting powers of $\beta \Lambda$ alone does not give a proper accounting of the relative magnitude of adjacent terms in the loop expansion.

\section{Appendix B.2. Bridge diagrams}

Bridge diagrams are more complicated to handle. We proceed in two steps, first arguing that a similar phase-space suppression means that only the one-loop bridge need be considered, and not multi-loop or rainbow bridges. In a second step, we argue that chains of bridges can be ignored because they make a contribution at leading order in powers of $\beta \Lambda$ which is precisely momentum-independent. A contribution of this type can be absorbed into coupling constants and becomes unobservable. The one-loop bridge is itself momentum-independent at leading order in powers of $\beta \Lambda$, so that the only contributions at this order which are not suppressed by a phase space factor of order $\sim 100$ are the oblique corrections considered in \$3. It will transpire that we expect corrections to the purely oblique analysis of $\S \S 3$ to occur at a relative order of roughly 
$1 / 8 \pi^{2} \approx 0.013$ or better. We believe this is an acceptable precision at which to predict what can be observed at present and future particle colliders. It is again important that there is a universal fermion coupling function, in order that the effective Fermi constant $G_{F}$ does not become species-dependent.

First consider a multi-loop contribution to the vacuum polarization of any $S U(2)$ gauge boson. At momentum transfer $q$, a calculation similar to those presented in $\$ 4$ establishes that this can be represented in the form

$$
\begin{aligned}
\Pi_{a b}^{n} \supseteq \frac{\bar{B}_{n}^{2} \beta^{2 n}}{\bar{B}_{1} n !} & -\mathrm{i})^{n} \int \frac{\mathrm{d}^{4} \ell}{(2 \pi)^{4}} \frac{\mathrm{d}^{4} r_{1}}{(2 \pi)^{4}} \cdots \frac{\mathrm{d}^{4} r_{n-1}}{(2 \pi)^{4}} \\
& \times \frac{P_{a b}(\ell, q)}{\ell^{2}+M_{W}^{2}-\mathrm{i} \epsilon} \frac{1}{r_{1}^{2}+M_{\chi}^{2}-\mathrm{i} \epsilon} \cdots \frac{1}{r_{n-1}^{2}+M_{\chi}^{2}-\mathrm{i} \epsilon} \frac{1}{R^{2}+M_{\chi}^{2}-\mathrm{i} \epsilon},
\end{aligned}
$$

where $R=q+\ell+\sum_{j=1}^{n-1} r_{j}, \ell$ is the momentum carried by the exchanged gauge boson, and $P_{a b}(\ell, q)$ satisfies

$P_{a b} \equiv \eta_{a b}\left(\ell \cdot q-\gamma_{n} M_{W}^{2}\right)^{2}-\left(\ell_{a} q_{b}+\ell_{b} q_{a}\right)\left(\ell \cdot q-\gamma_{n} M_{W}^{2}\right)+\ell_{a} \ell_{b}\left(q^{2}+\gamma_{n}^{2} M_{W}^{2}\right)$.

Eq. (B.7) is suppressed by $2 n$ powers of the coupling $\beta$, but each scalar integral can contribute a power of $\Lambda^{2}$. Since $P_{a b} \sim \ell^{2}$, the $\ell$ integration can contribute terms of order $\Lambda^{4}$. This would seem to imply that terms of order $\Lambda^{2}(\beta \Lambda)^{2 n}$ could be present in the answer, but the correct conclusion depends on the relative magnitude of $R^{2}$. Unlike the daisy diagrams or one-loop bridges, there can be some regions of phase space where $\ell \sim r_{j} \sim \Lambda$ and $\left|\ell+\sum_{j=1}^{n-1} r_{j}\right| \sim 0$, so that $R \sim q$. However, if the region of phase space in which this finely-tuned cancellation occurs shrinks with increasing cutoff faster than $\Lambda^{2}$ then we can estimate the leading contribution by setting $R \sim \Lambda$ in Eq. (B.7). In practice, the enhanced region of phase space is negligibly small.

The $n$-loop bridge. To estimate the contribution of the $n$-loop bridge, we set $R \sim n \Lambda$ and replace each factor such as $\mathrm{d}^{4} r_{j} /(2 \pi)^{4}$ by $\Lambda^{2} / 8 \pi^{2}$. This choice for $R$ is tantamount to assuming that the $r_{j}$ and $\ell$ are randomly oriented, so that their cross terms approximately average to zero. This is likely to be a good approximation for large $n$ but may fail for $n \sim \mathrm{O}(1)$, so we will demonstrate explicitly that this procedure gives the correct answer for the 2-loop bridge. We consider this to be reasonable evidence that our estimate is reliable for all $n$. Proceeding in this way, it follows that the $n$-loop bridge makes a contribution to the vacuum polarization which is roughly equal to

$$
\Pi_{a b}^{n} \supseteq \frac{1}{4} \eta_{a b}\left(2 q^{2}+\gamma_{n}^{2} M_{W}^{2}\right) \frac{\bar{B}_{n}^{2}(\beta \Lambda)^{2 n}}{\bar{B}_{1} n \cdot n !}\left(\frac{1}{8 \pi^{2}}\right)^{n} .
$$

For the special case of an exponential coupling-for which $\bar{B}_{n}=1$ for all $n$-this can be resummed to give

$$
\Pi_{a b} \supseteq \frac{1}{4} \eta_{a b}\left(2 q^{2}+\gamma^{2} M_{W}^{2}\right)\left\{-\gamma_{E}+\operatorname{Ei}\left(\frac{\beta^{2} \Lambda^{2}}{8 \pi^{2}}\right)-\ln \frac{\beta^{2} \Lambda^{2}}{8 \pi^{2}}\right\},
$$

where $\gamma_{E} \approx 0.577$ is the Euler-Mascheroni constant and $\operatorname{Ei}(z)$ is the exponential integral. As in the case of the daisy diagrams this is dominated by its one-loop contribution, with the contribution of higher loops being suppressed by the phase-space factor $1 / 8 \pi^{2}$. 
Two-loop bridge. In the special case of the two-loop bridge, Eq. (B.7) can be evaluated by the method of Feynman parameters, as in $\$$, with the result that

$$
\begin{aligned}
\Pi_{a b}^{(2)}=\frac{\bar{B}_{2}^{2} \beta^{4}}{\bar{B}} & \left(\frac{1}{8 \pi^{2}}\right)^{2} \int_{0}^{1} \mathrm{~d} x \mathrm{~d} y \mathrm{~d} z \delta(1-x-y-z) \\
& \times \int_{0}^{\Lambda} \ell^{3} \mathrm{~d} \ell \int_{0}^{\Lambda} r^{3} \mathrm{~d} r \frac{\frac{1}{4} \ell^{2}\left(2 q^{2}+\gamma_{2}^{2} M_{W}^{2}\right)+\left(X q^{2}+\gamma_{2} M_{W}^{2}\right)^{2}}{\Gamma^{3}},
\end{aligned}
$$

where $\Gamma$ is defined by

$$
\Gamma \equiv(1-x) r^{2}+W \ell^{2}+X Y q^{2}+x M_{W}^{2}+(1-x) M_{\chi}^{2}
$$

and the three quantities $W, X$ and $Y$ satisfy

$$
\begin{aligned}
W & \equiv \frac{(1-x)(1-y)-z^{2}}{1-x}, \\
X & \equiv \frac{z(1-z)}{(1-x)(1-y)-z^{2}}, \\
Y & \equiv \frac{(1-x)(1-y)-z^{2}+z(1-z)}{1-x} .
\end{aligned}
$$

Performing the $\ell$ and $r$ integrals and keeping only the leading term in powers of $\beta \Lambda$, this vacuum polarization can be simplified to read

$$
\begin{gathered}
\Pi_{a b}^{(2)} \simeq-\frac{\bar{B}_{2}^{2}(\beta \Lambda)^{4}}{\bar{B}}\left(2 q^{2}+\gamma_{2}^{2} M_{W}^{2}\right) \frac{1}{32}\left(\frac{1}{8 \pi^{2}}\right)^{2} \int_{0}^{1} \mathrm{~d} x \mathrm{~d} y \mathrm{~d} x \delta(1-x-y-z) \\
\times \frac{1}{W^{3}(W+1-x)}\left[W+(W+1-x) \ln \frac{1-x}{W+1-x}\right],
\end{gathered}
$$

where by " $\simeq$ " we mean that this relationship is true up to terms of order $\beta^{2}(\beta \Lambda)^{2}$ which we have neglected. The possibility of enhanced regions of phase space where the loop momenta approximately cancel to leave an anomalously small propagator $\sim 1 / q^{2}$ (rather than $\sim 1 / \Lambda^{2}$ ) has been replaced by the potential for large contributions from the Feynman parameter integrals. Indeed, inspection of Eq. (B.11) shows when considering only the leading term in powers of $\Lambda$ we might find a divergence roughly like $\int \mathrm{d} x /(1-x)^{3}$. This would be finite when terms of all orders in $\Lambda$ were included, but would manifest as an apparent divergence in the truncated series. If such a divergence appears, it should be regulated at a scale roughly of order $M_{\chi}^{2} / \Lambda^{2}$ where other terms in the perturbation theory become important, allowing enhanced phase space regions to appear. However, when these integrals are treated sufficiently carefully we find that no divergences occur and therefore that no enhanced regions of phase space exist. The integral can be evaluated by an adaptive Monte Carlo technique, and we find its numerical value to be roughly $\approx 0.025$. We conclude that Eq. (B.15) is as small in magnitude, or slightly smaller, than our estimate Eq. (B.10).

Chains of bridges. Now consider chains of bridges. We will first give an argument that the leading term in powers of $\beta \Lambda$ is entirely momentum independent for a one-loop 

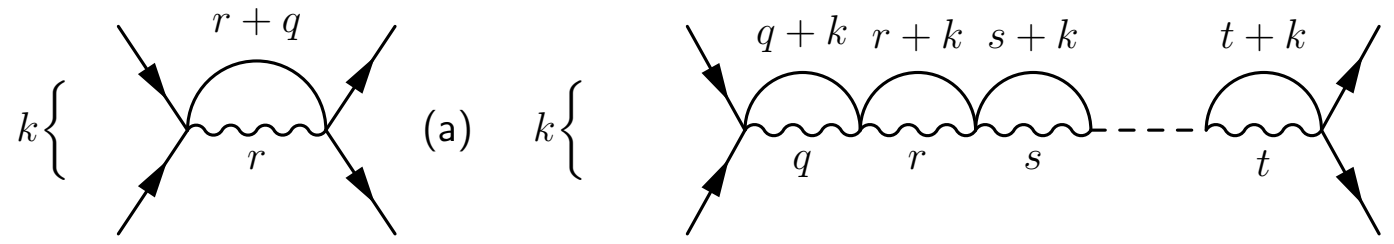

Figure B2. Bridge diagrams. In (a), $2 \rightarrow 2^{\prime}$ fermion scattering is dressed by a single dark energy bridge, in which a single dark energy particle is emitted or absorbed at the scattering vertices. In (b), a chain of bridges is exchanged. In principle, more complicated configurations exist in which the bridge is itself built out of rainbows of sub-bridges. We neglect these, since we anticipate that they will be suppressed by extra powers of the phase-space factor $1 / 8 \pi^{2}$.

bridge, before generalizing this to chains of arbitrary length. In each case, we use the results of the previous section to drop terms containing more than a single loop.

The single bridge is shown in Fig. B2(a). We assume that it describes a bridged fermion scattering process, between two fermion species which couple conformally to dark energy via the coupling function $F\left(\beta_{f} \chi\right)$ and coupling $\beta_{f}$. (The generalization to different couplings and coupling functions is obvious.) It is easy to see that the effect of the bridge is the same as inclusion of an extra term in the propagator, corresponding to

$$
\begin{aligned}
& \bar{F}_{1}^{2} \beta_{f}^{2} \int \frac{\mathrm{d}^{4} r}{(2 \pi)^{4}}(\left.\eta_{a b}+\frac{r_{a} r_{b}}{M_{W}^{2}}\right) \frac{1}{r^{2}+M_{W}^{2}-\mathrm{i} \epsilon} \frac{1}{(r+q)^{2}-\mathrm{i} \epsilon} \\
& \simeq \frac{\mathrm{i} \bar{F}_{1}^{2} \beta_{f}^{2}}{16 \pi^{2}} \eta_{a b} \int_{0}^{1} \mathrm{~d} x\left(-\frac{1}{2} \frac{\Lambda^{2}}{\Lambda^{2}+\Delta^{2}}+\frac{1}{2} \ln \left[1+\frac{\Lambda^{2}}{\Delta^{2}}\right]\right. \\
&\left.\quad+\frac{1}{8 M_{W}^{2}} \frac{\Lambda^{4}+2 \Lambda^{2} \Delta^{2}}{\Lambda^{2}+\Delta^{2}}-\frac{\Delta^{2}}{4 M_{W}^{2}} \ln \left[1+\frac{\Lambda^{2}}{\Delta^{2}}\right]\right),
\end{aligned}
$$

where $\Delta$ satisfies

$$
\Delta^{2}=x(1-x) q^{2}+x M_{\chi}^{2}+(1-x) M_{W}^{2} .
$$

Clearly, the leading term in powers of $\beta \Lambda$ is momentum independent.

The case of multiple bridges chained together is shown in Fig. B2(b). One may wonder why it is necessary to consider such chains, since we have already argued that diagrams with a large number of loops are suppressed by powers of the phase-space factor $8 \pi^{2}$. The reason is that to study corrections to the propagator, which potentially shift the location of its pole, one must resum enough diagrams to capture shifts in the physical mass of the particle. Such shifts are not captured at any finite order in perturbation theory.

Exchange of the chained bridge with $n$ internal vertices is equivalent to introducing an extra term in the propagator, which takes the form

$$
\begin{gathered}
\bar{F}_{1}^{2} \beta_{f}^{2}\left(\bar{B}_{2} \beta^{2}\right)^{n}(\mathrm{i})^{n} \int \frac{\mathrm{d}^{4} q}{(2 \pi)^{4}} \cdots \int \frac{\mathrm{d}^{4} t}{(2 \pi)^{4}} \frac{1}{q^{2}+M_{W}^{2}-\mathrm{i} \epsilon} \frac{1}{(q+k)^{2}+M_{\chi}^{2}-\mathrm{i} \epsilon} \cdots \\
\times\left(\eta_{a b}+\frac{q_{a} q_{b}}{M_{W}^{2}}\right)\left(\eta^{b c}\left[q \cdot r-\gamma_{2} M_{W}^{2}\right]-q^{c} r^{b}\right)
\end{gathered}
$$




$$
\begin{aligned}
& \times\left(\eta_{c d}+\frac{r_{c} r_{d}}{M_{W}^{2}}\right)\left(\eta^{d c}\left[r \cdot s-\gamma_{2} M_{W}^{2}\right]-r^{c} s^{d}\right) \\
& \times\left(\eta_{e f}+\frac{s_{e} s_{f}}{M_{W}^{2}}\right) \ldots \\
& \times\left(\eta_{g g}+\frac{t_{g} t_{h}}{M_{W}^{2}}\right) .
\end{aligned}
$$

In the first contraction, the cubic term $\sim q^{3}$ cancels out. In the second contraction, the cubic term $\sim r^{3}$ cancels out. The same sequence recurs throughout the chain. This implies that the only way to bring each momentum integral to order $\sim \Lambda^{2}$ is consider only the quadratic term from each propagator, which multiplies the momentum-independent quantity $\gamma_{W} M_{W}^{2}$. Accordingly, one can conclude that the resummed contribution at leading order in $\beta \Lambda$ behaves like an extra term in the propagator of the form

$$
\frac{\mathrm{i}}{64 \pi^{2}} \bar{F}_{1}^{2}\left(\beta_{f} \Lambda\right)^{2} \eta_{a b} \frac{1}{M_{W}^{2}} \frac{1}{1-\frac{\bar{B}_{2} \gamma_{2}[\beta \Lambda]^{2}}{64 \pi^{2}}} .
$$

Once again, this term is momentum-independent and contributes only to an unobservable shift - the same for all species - in the relevant masses and coupling constants.

\section{References}

[1] J. Khoury and A. Weltman, Chameleon fields: Awaiting surprises for tests of gravity in space, Phys. Rev. Lett. 93 (2004) 171104, arXiv:astro-ph/0309300.

[2] J. Khoury and A. Weltman, Chameleon cosmology, Phys. Rev. D69 (2004) 044026, arXiv:astro-ph/0309411.

[3] D. F. Mota and J. D. Barrow, Local and Global Variations of The Fine Structure Constant, Mon. Not. Roy. Astron. Soc. 349 (2004) 291, arXiv:astro-ph/0309273.

[4] T. Clifton, D. F. Mota, and J. D. Barrow, Inhomogeneous gravity, Mon. Not. Roy. Astron. Soc. 358 (2005) 601, arXiv:gr-qc/0406001.

[5] P. Brax, C. van de Bruck, A.-C. Davis, J. Khoury, and A. Weltman, Detecting dark energy in orbit: The cosmological chameleon, Phys. Rev. D70 (2004) 123518, arXiv:astro-ph/0408415.

[6] D. F. Mota and D. J. Shaw, Evading equivalence principle violations, astrophysical and cosmological constraints in scalar field theories with a strong coupling to matter, Phys. Rev. D75 (2007) 063501, arXiv:hep-ph/0608078.

[7] P. Brax, C. van de Bruck, and A.-C. Davis, Compatibility of the chameleon-field model with fifthforce experiments, cosmology, and PVLAS and CAST results, Phys. Rev. Lett. 99 (2007) 121103, arXiv:hep-ph/0703243.

[8] P. Brax, C. van de Bruck, A.-C. Davis, D. F. Mota, and D. J. Shaw, Testing Chameleon Theories with Light Propagating through a Magnetic Field, Phys. Rev. D76 (2007) 085010, arXiv:0707.2801.

[9] P. Brax, C. van de Bruck, A.-C. Davis, D. F. Mota, and D. J. Shaw, Detecting Chameleons through Casimir Force Measurements, Phys. Rev. D76 (2007) 124034, arXiv:0709.2075.

[10] C. Burrage, Supernova Brightening from Chameleon-Photon Mixing, Phys. Rev. D77 (2008) 043009, arXiv:0711.2966.

[11] P. Brax, C. van de Bruck, A.-C. Davis, and D. J. Shaw, $f(R)$ Gravity and Chameleon Theories, arXiv:0806.3415.

[12] A. S. Chou et al., A Search for chameleon particles using a photon regeneration technique, arXiv:0806.2438. 
[13] H. Gies, D. F. Mota, and D. J. Shaw, Hidden in the Light: Magnetically Induced Afterglow from Trapped Chameleon Fields, Phys. Rev. D77 (2008) 025016, arXiv:0710.1556.

[14] M. Ahlers, A. Lindner, A. Ringwald, L. Schrempp, and C. Weniger, Alpenglow - A Signature for Chameleons in Axion-Like Particle Search Experiments, Phys. Rev. D77 (2008) 015018, arXiv:0710.1555.

[15] C. Burrage, A.-C. Davis, and D. J. Shaw, Detecting Chameleons: The Astronomical Polarization Produced by Chameleon-like Scalar Fields, arXiv:0809.1763.

[16] M. Kleban and R. Rabadan, Collider bounds on pseudoscalars coupling to gauge bosons, arXiv:hep-ph/0510183.

[17] S. M. Carroll, Quintessence and the rest of the world, Phys. Rev. Lett. 81 (1998) 3067-3070, arXiv:astro-ph/9806099.

[18] D. J. Shaw, Charge non-conservation, dequantisation, and induced electric dipole moments in varying-alpha theories, Phys. Lett. B632 (2006) 105-108, arXiv:hep-th/0509093.

[19] M. B. Einhorn and J. Wudka, Screening of heavy scalars beyond the Standard Model, Phys. Rev. D47 (1993) 5029-5037.

[20] M. J. G. Veltman, The Screening theorem and the Higgs system, Acta Phys. Polon. B25 (1994) $1627-1636$.

[21] Particle Data Group Collaboration, C. Amsler et al., Review of particle physics, Phys. Lett. B667 (2008) 1.

[22] M. J. G. Veltman, Second Threshold in Weak Interactions, Acta Phys. Polon. B8 (1977) 475.

[23] M. J. G. Veltman, Limit on Mass Differences in the Weinberg Model, Nucl. Phys. B123 (1977) 89.

[24] J. van der Bij and M. J. G. Veltman, Two Loop Large Higgs Mass Correction to the $\rho$ Parameter, Nucl. Phys. B231 (1984) 205.

[25] M. B. Einhorn and J. Wudka, Screening of heavy Higgs radiative effects, Phys. Rev. D39 (1989) 2758.

[26] P. Sikivie, L. Susskind, M. B. Voloshin, and V. I. Zakharov, Isospin Breaking in Technicolor Models, Nucl. Phys. B173 (1980) 189.

[27] C. P. Burgess and D. London, On anomalous gauge boson couplings and loop calculations, Phys. Rev. Lett. 69 (1992) 3428-3431.

[28] C. P. Burgess and D. London, Uses and abuses of effective Lagrangians, Phys. Rev. D48 (1993) 4337-4351, arXiv:hep-ph/9203216.

[29] J. M. Cornwall, D. N. Levin, and G. Tiktopoulos, Derivation of Gauge Invariance from High-Energy Unitarity Bounds on the s Matrix, Phys. Rev. D10 (1974) 1145.

[30] M. S. Chanowitz, M. Golden, and H. Georgi, Low-Energy Theorems for Strongly Interacting W's and Z's, Phys. Rev. D36 (1987) 1490.

[31] S. Donnachie, H. G. Dosch, O. Nachtmann, and P. Landshoff, Pomeron physics and QCD, vol. 19 of Camb. Monogr. Part. Phys. Nucl. Phys. Cosmol. Cambridge, UK, 2002. 347pp.

[32] G. Dissertori, I. G. Knowles, and M. Schmelling, High energy experiments and theory. Oxford, Clarendon, UK, 2003. 538pp.

[33] M. E. Peskin and T. Takeuchi, A New constraint on a strongly interacting Higgs sector, Phys. Rev. Lett. 65 (1990) 964-967.

[34] M. E. Peskin and T. Takeuchi, Estimation of oblique electroweak corrections, Phys. Rev. D46 (1992) 381-409.

[35] G. Altarelli and R. Barbieri, Vacuum polarization effects of new physics on electroweak processes, Phys. Lett. B253 (1991) 161-167.

[36] G. Altarelli, R. Barbieri, and S. Jadach, Toward a model independent analysis of electroweak data, Nucl. Phys. B369 (1992) 3-32.

[37] I. Maksymyk, C. P. Burgess, and D. London, Beyond S, T and U, Phys. Rev. D50 (1994) 529-535, arXiv:hep-ph/9306267.

[38] C. P. Burgess, S. Godfrey, H. Konig, D. London, and I. Maksymyk, A Global fit to extended 
oblique parameters, Phys. Lett. B326 (1994) 276-281, arXiv:hep-ph/9307337.

[39] A. Kundu and P. Roy, A General Treatment of Oblique Parameters, Int. J. Mod. Phys. A12 (1997) 1511-1530, arXiv:hep-ph/9603323.

[40] J. L. Rosner, M. P. Worah, and T. Takeuchi, Oblique corrections to the $W$ width, Phys. Rev. D49 (1994) 1363-1369, arXiv:hep-ph/9309307.

[41] H. Lehmann, K. Symanzik, and W. Zimmermann, On the formulation of quantized field theories, Nuovo Cim. 1 (1955) 205-225.

[42] CAST Collaboration, S. Andriamonje et al., An improved limit on the axion-photon coupling from the CAST experiment, JCAP 0704 (2007) 010, arXiv:hep-ex/0702006.

[43] A. Afanasev et al., New Experimental limit on Optical Photon Coupling to Neutral, Scalar Bosons, Phys. Rev. Lett. 101 (2008) 120401, arXiv:0806.2631.

[44] G. Weiglein, Electroweak Physics at the ILC, J. Phys. Conf. Ser. 110 (2008) 042033, arXiv:0711.3003.

[45] C. Burrage, A.-C. Davis, and D. J. Shaw, Active Galactic Nuclei Shed Light on Axion-likeParticles, arXiv:0902.2320.

[46] D. Seery, Magnetogenesis and the primordial non-gaussianity, arXiv:0810.1617.

[47] B. J. Carr and J. E. Lidsey, Primordial black holes and generalized constraints on chaotic inflation, Phys. Rev. D48 (1993) 543-553.

[48] T. P. Cheng and L. F. Li, Gauge theory of elementary particle physics. Oxford, Clarendon, UK, 1984. 536pp. (Oxford Science Publications). 